\title{
Low-frequency waves in the Earth's magnetosheath: present status
}

\author{
S. J. Schwartz, D. Burgess, J. J. Moses \\ Astronomy Unit, Queen Mary and Westfield College, Mile End Road, London E1 4NS, United Kingdom \\ Received: 31 January 1996/Revised: 30 April 1996/Accepted: 29 May 1996
}

\begin{abstract}
The terrestrial magnetosheath contains a rich variety of low-frequency ( $\lesssim$ proton gyrofrequency) fluctuations. Kinetic and fluid-like processes at the bow shock, within the magnetosheath plasma, and at the magnetopause all provide sources of wave energy. The dominance of kinetic features such as temperature anisotropies, coupled with the high- $\beta$ conditions, complicates the wave dispersion and variety of instabilities to the point where mode identification is difficult. We review here the observed fluctuations and attempts to identify the dominant modes, along with the identification tools. Alfvén/ioncyclotron and mirror modes are generated by $T_{\perp} / T_{\|}>1$ temperature anisotropies and dominate when the plasma $\beta$ is low or high, respectively. Slow modes may also be present within a transition layer close to the subsolar magnetopause, although they are expected to suffer strong damping. All mode identifications are based on linearized theory in a homogeneous plasma and there are clear indications, in both the data and in numerical simulations, that nonlinearity and/or inhomogeneity modify even the most basic aspects of some modes. Additionally, the determination of the wave vector remains an outstanding observational issue which, perhaps, the Cluster mission will overcome.
\end{abstract}

\section{Introduction}

The interaction of the super-magnetosonic solar wind flow with the obstacle of the Earth's magnetic field results in the formation of a magnetospheric cavity bounded by the magnetopause and a bow shock upstream thereof. The layer in between these two surfaces, known as the magnetosheath, enables the incident solar wind flow, reduced to sub-magnetosonic speeds by the shock, to be diverted around the magnetosphere. The magnetosheath is a re-

Correspondence to: S. J. Schwartz gion rich in wave modes which in turn carry information and redistribute energy and momentum from the bow shock to the magnetopause and vice versa. Since it is the magnetosheath which ultimately delivers solar wind material to the magnetosphere, the extent to which the magnetosheath modifies the solar plasma has important implications for the basic solar-terrestrial interaction.

Several aspects of the magnetosheath complicate our understanding. The dissipation provided by the bow shock results in high kinetic temperatures, so that the plasma $\beta$ is large and the magnetic field is unable to order the plasma into a nearly laminar flow. It is a collisionless plasma, so that anisotropies, particle beams, unequal species temperatures, and other features are preserved unless collisionless processes, such as wave-particle scattering, are able to bring the plasma toward equilibrium. It is a non-uniform medium with substantial gradients over scales from thousands of kilometers or less (corresponding to foreshock-related turbulence) to several Earth radii (corresponding to the distance between the bow shock and magnetopause) or longer (corresponding to changes along the bow shock from quasi-perpendicular to quasiparallel orientations of the interplanetary magnetic field with respect to the local bow shock normal). It is observed to be highly turbulent; that is, $O(1)$ fluctuations in magnetic field, density, and bulk flow velocity are usually present over a broad range of frequencies (see also Lacombe and Belmont, 1995; Hubert, 1994; Omidi et al., 1994; Onsager and Thomsen, 1991; Fazakerley and Southwood, 1994b).

In this study, we present an overview of the present understanding of low frequency (below the proton gyrofrequency) wave modes and instabilities. We shall concentrate on the sources of such waves and the methods of mode identification which have been made to date. We shall see that despite significant efforts in recent years, some uncertainty remains. We shall not address here higher frequency modes, such as "lion roars" (Tsurutani et al., 1982), and standing structures, e.g., the plasma depletion layer (Zwan and Wolf, 1976), slow mode transition (Song et al., 1992b; Song et al., 1993; Song, 1994), and 
rotational discontinuities which are also found in the magnetosheath. Additionally, transient processes at the bow shock (e.g., hot flow anomalies, see Schwartz et al., 1988; Thomsen et al., 1988a; Paschmann et al., 1988; Schwartz, 1995) and at the magnetopause (e.g., flux transfer events, Russell and Elphic, 1978; Farrugia et al., 1988 and surface waves e.g., Sibeck et al., 1989; Sibeck, 1992) give rise to transient fluctuations in the magnetosheath.

In the next section, we give an overview of the main postulated sources of magnetosheath waves and their basic observational characteristics. We then turn to a discussion of mode identification via various linear theories and derived tools. We study in more detail the observations and identification of the Alfvén/ion-cyclotron, mirror modes, and slow/sound waves. Our discussion section includes recommendations and requirements to advance this subject further.

For further reference, we define below the various quantities and symbols used in this study. SI units are used throughout.

\begin{tabular}{|c|c|c|}
\hline Symbol & Formula & Quantity \\
\hline$\vec{B}$ & & Magnetic field \\
\hline$\vec{A}$ & & $\begin{array}{l}\text { Vector potential (Coulomb } \\
\text { gauge } \nabla \cdot \vec{A}=0 \text { ) }\end{array}$ \\
\hline$T$ & & Temperature \\
\hline$P$ & & Pressure \\
\hline$P_{B}$ & $B^{2} / 2 \mu_{\mathrm{o}}$ & Magnetic pressure \\
\hline$n$ & & Number density \\
\hline$\rho$ & & Mass density \\
\hline$\vec{V}$ & & Bulk velocity \\
\hline$\beta$ & $P /\left(B^{2} / 2 \mu_{0}\right)$ & Plasma beta \\
\hline "o" & & $\begin{array}{l}\text { Average or background } \\
\text { values }\end{array}$ \\
\hline “\|” $[“ \perp ”]$ & & $\begin{array}{l}\text { Component along } \\
\text { [perpendicular to }\rceil \vec{B}\end{array}$ \\
\hline “ $j "$ & & Species "j" only \\
\hline$v_{A}$ & $B_{\mathrm{o}} / \sqrt{\mu_{\mathrm{o}} \rho_{\mathrm{o}}}$ & Alfvén speed \\
\hline c & & Fluctuating component \\
\hline$\omega$ & & Wave angular frequency \\
\hline$\vec{k}$ & & Wave vector \\
\hline$\omega_{r}[\gamma]$ & $\operatorname{Re} \omega[\operatorname{Im} \omega]$ & Real frequency [growth rat \\
\hline & $q B / m$ & Cyclotron frequency \\
\hline$\omega_{p}$ & $\sqrt{n q^{2} / m \varepsilon_{0}}$ & Plasma frequency \\
\hline
\end{tabular}

\section{Magnetosheath waves: sources and observational overview}

There are several candidate sources for fluctuations observed in the magnetosheath. These range from processes upstream of or at the bow shock (including the ambient solar wind turbulence) to local processes within the magnetosheath to the magnetopause itself. Local wave generation/amplification occurs due to non-Maxwellian features of the particle distribution (later we discuss various fluid and kinetic descriptions of wave modes). Such kinetic features are often products or remnants of processes occurring more remotely, e.g., at the bow shock. It is also possible to detect systematic trends in both the waves and particles through the magnetosheath due to wave propagation and generation mechanisms which dominate under different regimes (e.g., low $\beta$ versus high $\beta$ ) and to the macroscopic magnetosheath draping and flow diversion about the magnetosphere. Such trends help confirm, by inference, the operation of particular local wave generation mechanisms. Krauss-Varban (1994) reviews the general problem of mode transmission and coupling through the bow shock and inhomogeneous magnetosheath plasma. Other general studies and reviews are also helpful (Hubert, 1994; Lacombe and Belmont, 1995; Omidi et al., 1994; Onsager and Thomsen, 1991).

\subsection{Reflected-gyrating ions at the quasi-perpendicular shock}

Super-critical collisionless fast magnetosonic shock waves, such as large portions of the Earth's bow shock, for which the angle between the upstream magnetic field and the shock normal is $>45^{\circ}$, dissipate the incident bulk flow energy primarily via a coherent reflection process in which a fraction of the incident ion population is turned around at the main shock "ramp." In this geometry, such reflected ions gyrate around the upstream field and return to the ramp because of their guiding centre motion. At this second encounter they pass into the downstream region where, in this idealized picture, they form a partially filled ring beam distribution, which gyrates around the initially transmitted core population (Paschmann et al., 1982; Sckopke et al., 1983; Gosling and Robson, 1985).

There are two consequences of this behaviour. Firstly, the mutual gyration of the core and reflected components in the downstream region establishes a spatially coherent overshoot, undershoot and successive oscillations about the mean downstream field. This nonlinear structure, which stands in the downstream flow and is attached to the ramp, imposes a non-uniform background state which any subsequent instability must grow on and/or compete with. Secondly, the combined core/ring-beam distribution possesses an effective temperature anisotropy with $T_{\perp} / T_{\|}>1$ which is unstable to Alfvén/ion-cyclotron waves under low $\beta$ conditions and to mirror modes at high $\beta$ (Gary et al., 1993b; Lacombe et al., 1990; Lacombe et al., 1992; McKean et al., 1995). As we move toward the magnetopause, the imposed structure, waves and instabilities evolve.

\subsection{Quasi-parallel shock}

Under quasi-parallel geometries, when the upstream field in within $\sim 45^{\circ}$ of the shock normal, particles traverse the shock in both directions, with the guiding centre motion of any reflected ions now directed upstream. The result is an extended foreshock region with populations of energetic "diffuse" ions and large amplitude ULF turbulence $\left(\delta B / B_{0} \gtrsim 1\right)$. The turbulence interacts with, and accelerates, the energetic ions in the foreshock via both right-handed and left-handed instabilities, but is ultimately convected toward the shock by the super-magnetosonic solar wind 
flow. Such trains of short "pulsations" (Greenstadt et al., 1977; Thomsen et al., 1990b) or short large amplitude magnetic structures (SLAMS) (Schwartz, 1991) mask or replace a distinct shock transition, at least under some circumstances (Thomsen et al., 1988b; Schwartz and Burgess, 1991). Under such conditions, the magnetosheath, like the quasi-parallel foreshock and shock, becomes a patchworked structure of dense warm regions (of plasma which has not suffered significant heating due to ion reflection from transient quasi-perpendicular fields) and less dense hot regions (heated via ion reflection and strong beam-plasma coupling) (Gosling et al., 1989; Thomsen et al., 1988b; Thomsen et al., 1990a; Schwartz and Burgess, 1991). This pattern persists well downstream of the quasi-parallel shock, suggesting that the variations seen here are merely the imposed spatial structure formed upstream/near the shock transition. Fuselier et al. (1994), however, report spectral features and an inverse temperature anisotropy/beta correlation in the quasi-parallel magnetosheath which is similar to that found under quasiperpendicular geometries, suggesting similar local processes and wave generation in both cases.

\subsection{The magnetopause itself}

Ultimately it is the magnetopause which drives the bow shock, and which therefore must influence the magnetosheath through which magnetopause signals are carried upstream. In simple MHD terms, one would expect three transitions, corresponding to the fast mode (e.g., the bow shock), intermediate mode/rotational discontinuity, and slow mode all standing sequentially at the points in the magnetosheath flow, which progressively decelerates from the super-fast magnetosonic solar wind flow down to sub-slow magnetosonic speeds near the sub-solar magnetopause. Each such nonlinear front can amplify or modify the waves passing through it. There is some evidence for a standing slow mode structure (Song et al., 1992a). The detection of slow mode waves, which should be heavily Landau damped in the high $\beta$ magnetosheath plasma, downstream of this transition is less conclusive at this stage. No one has reported to date any analogous fast mode waves propagating from the magnetopause to the fast mode bow shock, although the isotropic rather than guided nature of the fast mode does not lend itself to easily identifiable characteristics in the turbulent magnetosheath.

Additionally, near the sub-solar portion of the magnetopause the magnetic field lines drape around the nose of the obstacle. This results in perpendicular compression of the field, a depletion of the total plasma density, and a temperature anisotropy $T_{\perp}>T_{\|}$(Crooker et al., 1979; Anderson and Fuselier, 1993; Zwan and Wolf, 1976). Thus the temperature anisotropy in this "plasma depletion layer" can drive Alfvén/ion-cyclotron or mirror instabilities. Finally, the magnetosheath flow interacts with the magnetopause via magnetic reconnection and via a generally sheared flow (e.g., on the flanks of the magnetopause) resulting in sources of surface waves, Kelvin-Helmholtz instabilities, or other disturbances there.

\subsection{Magnetosheath processes}

In performing its role of slowing and diverting the incident solar wind flow about the magnetosphere, the magnetosheath imposes systematic gradients in field and plasma parameters, as recent superposed epoch analyses reveal (Phan et al., 1994; Hill et al., 1995). The field line draping and consequent compression perpendicular to $\vec{B}$ operates throughout the magnetosheath to drive or maintain a $T_{\perp} / T_{\|}>1$ anisotropy, although only near the sub-solar magnetopause is the effect dramatic enough to warrant the distinction of a separately named region (the depletion layer discussed earlier). As we have seen, these anisotropies drive local instabilities and/or enhance existing fluctuations. Moreover, structures in the magnetosheath, e.g., a rotational discontinuity, can amplify or modify the fluctuations passing through it (Omidi and Winske, 1995; see also Fig. 11 later).

\subsection{Observational overview}

As a brief introduction to the observed fluctuations, Fig. 1 shows average power spectra for five categories of turbulence downstream of the quasi-perpendicular shock. Although categorized on the basis of magnetic spectral characteristics, these spectra also represent a transition from high $\beta$, low anisotropy $\left(T_{\perp} / T_{\|} \lesssim 1.5\right)$ (upper left plot) to low $\beta$, high anisotropy $\left(T_{\perp} / T_{\|} \sim 5\right)$ regimes (lower right). At high $\beta$ the fluctuations extend smoothly to low frequencies and are predominantly compressive; that is, the parallel field component contains most of the wave power. As we progress to lower $\beta$, higher anisotropy conditions, the transverse components become dominant, with the left-hand component systematically larger, and spectral features/peaks appear below frequencies corresponding to the proton and alpha particle gyrofrequencies. This progression with $\beta$ may be a simple consequence of linear plasma instability theory (Denton et al., 1994b) (see Sects. 5 and 6). The final set of spectra (bottom right) show two peaks, which are probably indicative of the simultaneous presence of alpha and proton Alfvén/ion-cyclotron modes. Denton et al. (1995) have gone on to consider the characteristic plasma variations for fluctuations near the sub-solar magnetopause. Systematic studies of wave/ plasma variations within the magnetosheath, however, have not been carried out, although isolated case studies have been performed (Lacombe et al., 1995; Leckband et al., 1995; Song et al., 1992a, b).

\section{Wave modes}

The interpretation of observed fluctuations in the magnetosheath requires a theoretical framework on which the observations can be placed. The subject of waves in a plasma, particularly a high $\beta$ one such as the magnetosheath, is complex, even to the level of serious nomenclature problems (Krauss-Varban et al., 1994). Moreover, almost all our theoretical understanding of waves in plasmas is based on linearized theory of some 


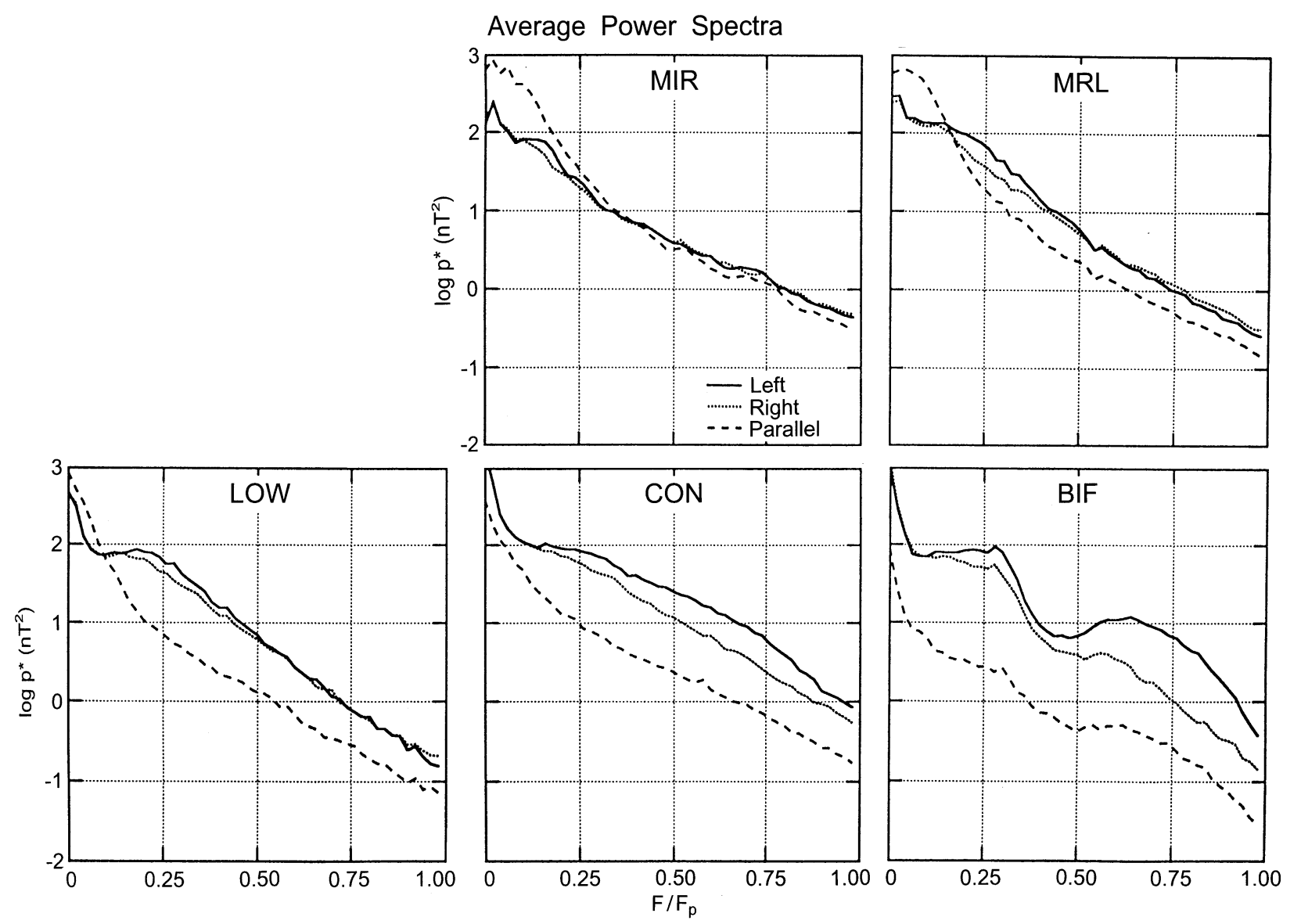

Fig. 1. Classes of magnetic power spectra found within the magnetosheath. The top left MIR spectra show predominantly parallel (i.e., compressive) fluctuations peaking at low frequencies and identified as mirror modes. There is a steady progression to the bottom right panel which is dominated by two higher frequency left-hand polarized peaks corresponding to alpha particle and proton cyclo-

sort in a homogeneous infinite medium. By contrast, the magnetosheath is a highly inhomogeneous medium with significant influences of its bounding regions and imposed structure on several scales. Finally, while the rather long length and time scales of the vast majority of the fluctuations suggest that fluid-like theories should be adequate to describe them, we know that there are kinetic effects which play important roles. Thus the most basic of questions, e.g., "Do a particular set of fluctuations correspond to mirror modes or slow modes?" poses several potential pitfalls in reaching an answer. We thus begin here by describing the wave modes found via different theoretical frameworks.

\subsection{Isotropic MHD waves}

Linearized ideal isotropic MHD theory admits three wellknown propagating wave solutions. All are linearly dispersive, that is the phase velocity is independent of the wavelength. There is also a fourth zero-frequency mode, referred to as an entropy mode, which is often ignored. The tron modes. The progression also proceeds, though not by any selection criteria, from high- $\beta$, low $T_{\perp} / T_{\|}(\lesssim 1.5)$ to low- $\beta$, high $T_{\perp} / T_{\|}(\sim 5)$ conditions and thus suggests the operation of the mirror and Alfvén/ion-cyclotron instabilities under their respectively appropriate conditions. (Fig. 5 of Anderson et al., 1994)

entropy mode represents a spatially structured medium in static equilibrium.

The intermediate/Alfven wave is a non-compressive mode which is guided along the magnetic field; i.e., its group velocity is field-aligned. The velocity and magnetic field perturbations are parallel (or anti-parallel) to one another and are linearly polarized in the transverse direction, i.e., along $\vec{k} \times \vec{B}_{\mathrm{o}}$. The oscillations are supported by the magnetic tension, leading to a phase velocity whose component along $\vec{B}_{\mathrm{o}}$ is the Alfvén speed $v_{A}$.

The other two MHD modes are polarized in the $\vec{k}-\vec{B}_{\text {o }}$ plane. The fast magnetosonic mode is compressive, with both magnetic and thermal pressure working in phase to provide the restoring force which supports the wave. This mode is nearly isotropic in the sense that the phase speed varies only weakly with direction, being largest at propagation perpendicular to $\vec{B}_{\mathrm{o}}$. By contrast, the slow magnetosonic mode is strongly guided by the field direction and is supported by a competition between the magnetic and thermal pressures. Accordingly, the density and field magnitude fluctuations are in anti-phase with one another. 
The three modes of ideal MHD are always ordered in phase speeds with the intermediate/Alfven wave lying between the fast and slow magnetosonic waves. Finally, it is worth bearing in mind that these ideal MHD modes are all purely oscillatory; there are no instabilities in linearized, homogeneous ideal MHD theory.

\subsection{Anisotropic MHD waves}

We have seen earlier that the magnetosheath plasma is not isotropic, and thus the application of isotropic MHD theory may be inadequate. Some improvement may be gained by including two equations of state, e.g., via CGL theory (Chew et al., 1956), to describe the plasma pressure parallel and perpendicular to the field. The result is a set of equations whose linearized forms yield three wave modes (Abraham-Shrauner, 1967) (more recently generalized to double polytropic equations of state (Hau and Sonnerup, 1993)) which are the counterparts of the three isotropic MHD modes. The Alfvén wave remains transverse but becomes the firehose instability for $P_{\mathrm{o} \|}>P_{\mathrm{o} \perp}+B_{\mathrm{o}}^{2} / \mu_{\mathrm{o}}$. As in isotropic MHD, the square of the wave frequencies are always real, so the firehose instability is non-oscillatory and grows with time. The fast magnetosonic wave behaves very similarly to its isotropic MHD namesake. Finally, the slow magnetosonic mode becomes the mirror instability for anisotropies satisfying

$\frac{P_{\mathrm{o} \perp}}{P_{\mathrm{o} \|}}>6\left(1+\frac{B_{\mathrm{o}}^{2}}{2 \mu_{\mathrm{o}} P_{\mathrm{o} \perp}}\right)$

The lowest threshold corresponds to wave-vectors perpendicular to the background magnetic field.

Interestingly, although it is possible to recover the isotropic MHD equations (with $\gamma=5 / 3$ ) from the CGL set by letting $P_{\perp}=P_{\|}$(Denton et al., 1994a), this is equivalent to introducing a process which instantaneously redistributes energy between the perpendicular and parallel degrees of freedom. As a consequence, the dynamics of superficially equivalent phenomena are different. For example, at propagation parallel to $\vec{B}_{0}$, the slow mode phase speed is $\sqrt{5 P_{\mathrm{o}} / 3 \rho_{\mathrm{o}}}$ in isotropic MHD and $\sqrt{3 P_{\mathrm{o} \|} \rho_{\mathrm{o}}}$ in CGL. Although in this case the CGL slow mode does induce perturbations in $P_{\perp}$, these have no dynamic role; that is, they do not enter the equation of motion.

\subsection{Two-fluid/Hall MHD waves}

Although the magnetosheath waves are generally dominated by low-frequency fluctuations, the power spectra (see Fig. 1) extend well above the proton cyclotron frequency. This is not surprising, as we have already seen that the sources of the waves are usually linked to distinct particle behaviour. Thus, the MHD assumption of frequencies well below the cyclotron frequency and wavelengths longer than a Larmor radius may not be appropriate. The next improvement to the wave theory is thus to retain finite Larmor radius effects via a two-fluid approach, treating the ions and electrons separately (Stringer, 1963). For waves dominated by the ions, a simplification can be achieved by ignoring the electron inertia, leading to the Hall MHD equations. See Krauss-Varban et al. (1994) for a review of two-fluid/Hall wave solutions, which are summarized in the left two panels of Fig. 2. The Hall MHD modes are always ordered in phase speed and can be simply related to their MHD counterparts at low frequencies. At higher frequencies, the finite Larmor radius/Hall corrections result in nonlinear dispersion, with the fast mode becoming positively dispersive (ultimately to join the whistler branch) and the slow mode negatively dispersive with a cutoff at the cyclotron frequency. The intermediate mode (Alfvén mode at low frequencies) becomes acoustic at high frequencies, and its dispersion at moderate frequencies is thus opposite at low and high $\beta$ in order to achieve a slope equal to the sound speed at high frequencies from its low-frequency beginnings.

Unfortunately, the advance represented by Hall MHD is made at the expense of re-introducing the assumption of isotropy. Moreover, as we shall see later, a more complete treatment of the particle effects yields different results. Thus, the added complexity of Hall MHD does not really improve the description of the wave modes in an accurate manner.

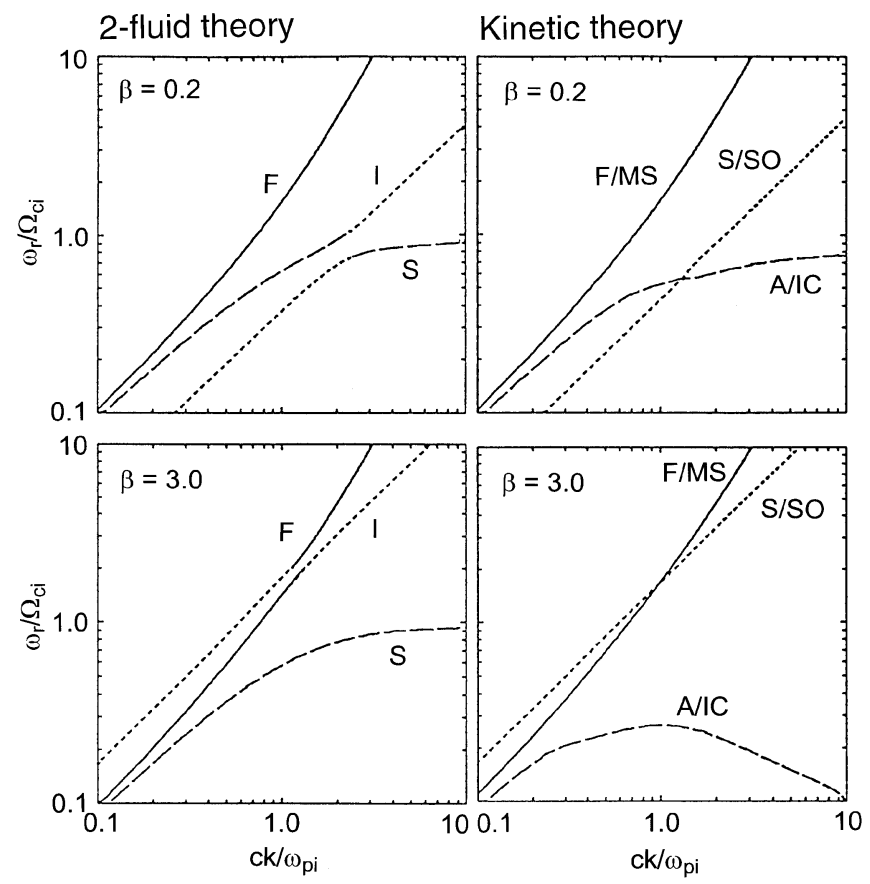

Fig. 2. Comparison of dispersion curves $\omega(k)$ for obliquely propagating waves $\left(\theta_{k B_{\mathrm{o}}}=30^{\circ}\right)$ as derived via two-fluid Hall MHD (left panels) and kinetic theory (right panels). At low $\beta$ (top panels) the two fluid fast $(F)$ mode corresponds to the kinetic fast magnetosonic $(F / M S)$ mode. The two-fluid intermediate mode $(I)$ behaves like the Alfvén/ion-cyclotron $(A / I C)$ at low frequencies and like the slow/sound $(S / S O)$ above the ion cyclotron frequency $\Omega_{c i}$. The twofluid slow mode $(S)$ corresponds more closely to the slow/sound $(S / S O)$ kinetic solution at low frequencies and to the Alfvén/ioncyclotron wave near $\omega_{r} \sim \Omega_{c i}$. At high $\beta$ (lower panels) none of the modes in two-fluid theory correspond to a single kinetic mode, (from Krauss-Varban et al., 1994, Fig. 1) 


\subsection{Kinetic/Vlasov waves and instabilities}

Kinetic theory readily takes into account multi-species plasmas, finite Larmor radii, temperature anisotropies, particle beams, and other phenomena which contribute to the plasma state of the magnetosheath. Although the linearized wave modes are more difficult to calculate (Stix 1992), widely available tools such as WHAMP (Rönmark, 1982) considerably ease the burden. Even in the relatively simple case of an isotropic proton-electron plasma at low frequencies the kinetic results deviate significantly from fluid treatments, as can be seen by comparing the left and right-hand panels of Fig. 2. In the kinetic treatment particles can exchange energy with the waves, giving rise to complex frequencies corresponding to wave growth or damping. Thus modes may cross in a diagram of Re $\omega$ versus $k$; the degeneracy is often removed in the complex plane. At high $\beta$, as typically found in the magnetosheath, the kinetic modes lose much of their resemblance to their fluid counterparts. The Alfvén/ion-cyclotron mode now becomes the slowest mode and actually shows real frequencies which decrease as the wave number is increased to large values, although the mode is heavily damped at these wave numbers. The fast mode, whose dispersion progresses smoothly toward the whistler regime, is slower at low frequencies than the 'slow/sound' mode. Additionally, kinetic theory admits a fourth wave mode which can be identified as the mirror mode (see Fig. 8 and Belmont $e t$ al., 1992). It has zero rest frame frequency and is lightly damped in a simple Maxwellian plasma, but can be unstable in a $\left(T_{\perp} / T_{\|}>1\right)$ plasma (see later) with a growth rate which confirms qualitatively but not quantitatively the CGL result in Eq. (1).

To make matters worse, the inclusion of multiple species, beams, anisotropies, etc., also introduces significant changes to the wave modes. At the most basic level, such kinetic deviations from thermal equilibrium can cause certain modes to become unstable and grow. Other growing modes, e.g., certain "beam modes", owe their existence to non-equilibrium conditions (Gary, 1993).

The sensitivity of kinetic modes to the zeroth order distribution functions has generated a whole industry of calculating dispersion and mode properties on the one hand, and added substantial complexity in the process of mode identification on the other. Before turning to this problem of identification, we draw here an obvious conclusion to the subject of linear wave theory: for most aspects of space plasmas, and certainly for the waves observed in the magnetosheath, fluid-based descriptions of any kind are inadequate, particularly under the $\beta>1$ conditions found there. And we have not yet considered complications due to inhomogeneity and nonlinearity!

\subsection{Mode identification}

To address the question of identifying a mode in the observations, let us take a step back and ask what a wave

Table 1. Transport ratios, polarizations, and other quantities for mode identification

\begin{tabular}{|c|c|c|c|}
\hline \multicolumn{4}{|c|}{ Quantities requiring only field information } \\
\hline Quantity & Symbol & Expression & Reference \\
\hline Magnetic compressibility & $C_{B}$ & $\frac{\left\langle\delta B_{\|} \delta B_{\|}\right\rangle_{\vec{k} \omega}}{\langle\delta \vec{B} \cdot \delta \vec{B}\rangle_{\vec{k} \omega}}$ & Denton et al. (1995) \\
\hline Non-coplanar ratio & $R_{n c}$ & $\frac{\left\langle\delta B_{y} \delta B_{y}\right\rangle_{\vec{k} \omega}}{\langle\delta \vec{B} \cdot \delta \vec{B}\rangle_{\vec{k} \omega}}$ & Denton et al. (1995) \\
\hline Transverse ratio & $T$ & $\frac{\langle\delta \vec{B} \cdot \delta \vec{B}\rangle_{\vec{k} \omega}-\left\langle\delta B_{\|} \delta B_{\|}\right\rangle_{\vec{k} \omega}}{\left\langle\delta B_{\|} \delta B_{\|}\right\rangle_{\vec{k} \omega}}$ & Song et al. (1994a) \\
\hline Magnetic compression ratio & $C_{B B}$ & $\frac{1}{\tan ^{2} \theta_{k B_{0}}} \frac{\left\langle\delta B_{\|} \delta B_{\|}\right\rangle_{\vec{k} \omega}}{\left\langle\delta B_{\perp} \delta B_{\perp}\right\rangle_{\vec{k} \omega}}$ & Krauss-Varban et al. (1994) \\
\hline Magnetic polarization $-B_{\mathrm{o}}$ & $P_{B, b_{\mathrm{o}}}$ & $\frac{\delta B_{y}}{i \delta B_{x}}$ & Krauss-Varban et al. (1994) \\
\hline Magnetic polarization $-k$ & $P_{B, k}$ & $\frac{i \delta B_{\xi}}{\delta B_{y}}$ & Krauss-Varban et al. (1994) \\
\hline Ellipticity & $\varepsilon_{B}$ & $\operatorname{Re}\left(\frac{\delta B_{\xi}-i \delta B_{y}}{\delta B_{\xi}+i \delta B_{y}}\right)$ & Krauss-Varban et al. (1994) \\
\hline Electric polarization $-B_{\mathrm{o}}$ & $P_{E, b_{\mathrm{O}}}$ & $\frac{i \delta E_{x}}{\delta E_{y}}$ & Krauss-Varban et al. (1994) \\
\hline & & $\frac{\langle\delta \vec{A} \cdot \delta \vec{B}\rangle_{k \omega}}{\langle\delta \vec{B} \cdot \delta \vec{B}\rangle_{\vec{k} \omega}}$ & Gary and Winske (1992) \\
\hline Helicity & $\sigma$ & $\frac{k^{2}}{\left|\omega^{2}\right|} \frac{\operatorname{Im}\left(\delta \vec{E} \cdot\left(\vec{k} \times \delta \vec{E}^{*}\right)\right)}{k \delta \vec{B}^{*} \cdot \delta \vec{B}}$ & Krauss-Varban et al. (1994) \\
\hline
\end{tabular}


Table 1. Continued

Quantities requiring plasma parameters

\begin{tabular}{|c|c|c|c|}
\hline Quantity & Symbol & Expression & Reference \\
\hline Density-field ratio & $C_{p j}$ & $\frac{\delta n_{j}}{n_{\mathrm{oj}}} \frac{B_{\mathrm{o}}}{\delta B_{\|}}$ & $\begin{array}{l}\text { Lacombe and Belmont (1995) } \\
\text { Krauss-Varban et al. (1994) }\end{array}$ \\
\hline Parallel compressibility & $C_{\| j} \equiv \operatorname{Re} C_{p j}$ & $\frac{\left\langle\delta n_{j} \delta B_{\|}\right\rangle_{\vec{k} \omega}}{n_{\mathrm{o} j} B_{\mathrm{o}}} \frac{B_{\mathrm{o}}^{2}}{\left\langle\delta B_{\|} \delta B_{\|}\right\rangle_{\vec{k} \omega}}$ & Gary and Winske (1992) \\
\hline Parallel phase ratio & $R_{\| n_{j}}$ or $C_{n B \| j}$ & $\frac{\left\langle\delta n_{j} \delta B_{\|}\right\rangle_{\vec{k} \omega}}{\sqrt{\left\langle\delta n_{j} \delta n_{j}\right\rangle_{k} \omega} \sqrt{\left\langle\delta B_{\|} \delta B_{\|}\right\rangle_{\vec{k} \omega}}}$ & $\begin{array}{l}\text { Denton et al. (1995) } \\
\text { Lacombe et al. (1992) }\end{array}$ \\
\hline Compressibility & $C_{j}$ & $\frac{\left\langle\delta n_{j} \delta n_{j}\right\rangle_{\vec{k} \omega}}{n_{\mathrm{o} j}^{2}} \frac{B_{\mathrm{o}}^{2}}{\langle\delta \vec{B} \cdot \delta \vec{B}\rangle_{\vec{k} \omega}}$ & Krauss-Varban et al. (1994) \\
\hline Alfvén ratio & $R_{A j}$ & $\frac{\left\langle\delta \vec{V}_{j} \cdot \delta \vec{V}_{j}\right\rangle_{\vec{k} \omega}}{v_{A}^{2}} \frac{B_{\mathrm{o}}^{2}}{\langle\delta \vec{B} \cdot \delta \vec{B}\rangle_{\vec{k} \omega}}$ & Denton et al. (1995) \\
\hline Compressional ratio & $C_{P j}$ & $\frac{\left\langle\delta P_{j} \delta P_{j}\right\rangle_{\vec{k} \omega}}{P_{\mathrm{o} j}^{2}} \frac{B_{\mathrm{o}}^{2}}{\langle\delta \vec{B} \cdot \delta \vec{B}\rangle_{\vec{k} \omega}}$ & Song et al. (1994a) \\
\hline Partition ratio & $P_{j}$ & $\frac{\delta P_{j}}{P_{\mathrm{o} j}} \frac{P_{B_{\mathrm{o}}}}{\delta P_{B}}$ & Song et al. (1994a) \\
\hline Doppler ratio & $D_{j}$ & $\frac{\left\langle\delta \vec{V}_{j} \cdot \delta \vec{V}_{j}\right\rangle_{\vec{k} \omega}}{V_{\mathrm{o}}^{2}} \frac{B_{\mathrm{o}}^{2}}{\langle\delta \vec{B} \cdot \delta \vec{B}\rangle_{\vec{k} \omega}}$ & Song et al. (1994a) \\
\hline Cross helicity & $\sigma_{c j}$ & $\frac{2}{v_{A} B_{\mathrm{o}}} \frac{\left\langle\delta \vec{V}_{j} \cdot \delta \vec{B}\right\rangle}{\frac{\left\langle\delta \vec{V}_{j} \cdot \delta \vec{V}_{j}\right\rangle_{\vec{k} \omega}}{v_{A}^{2}}+\frac{\langle\delta \vec{B} \cdot \delta \vec{B}\rangle_{\vec{k} \omega}}{B_{\mathrm{o}}^{2}}}$ & Gary and Winske (1992) \\
\hline
\end{tabular}

1. Here the Alfvén speed $v_{A}$ is calculated using only the proton mass density, i.e., $v_{A}=B_{\mathrm{o}} / \sqrt{\mu_{\mathrm{o}} \rho_{\mathrm{op}}}$

2. In this table, for any parameter $a$, the symbol $\delta a$ is the complex Fourier amplitude of the fluctuation, i.e., $a=a_{\mathrm{o}}+\delta a \exp i(\vec{k} \cdot \vec{x}-\omega t)$.

3. The angle bracket $\langle\delta a \delta b\rangle_{\vec{k} \omega}$ represents the Fourier transform of the correlation function $C_{a b}\left(\vec{x}, \vec{x}^{\prime}, t, t^{\prime}\right)$. In terms of the Fourier amplitudes, $\langle\delta a \delta b\rangle_{\vec{k} \omega}=\frac{1}{2}\left(\delta a^{*} \delta b+\delta b^{*} \delta a\right)$. See Gary and Winske (1992) Eqs. (1-4). These relations assume stationary and homogeneity

4. We have introduced the nomenclature "density-field ratio" and reverted to the symbol $C_{p j}$ used by Lacombe et al. (1992) to distinguish this quantity from the parallel compressibility, as in the past the same name and symbol have been used for both. Note that $C_{p j}$ is a complex quantity containing phase information (which is also accessible via $R_{\| n j}$ )

5. We have corrected/been informed of (D. Krauss-Varban, private communication) several misprints in Krauss-Varban et al. (1994). These include:

(a) The middle expressions in (A14) and (A15) should have $4 \pi \rho_{\mathrm{op}}$ instead of the appearance of $4 \pi \rho_{j}$; that is, the normalization (and Alfvén speed) is with respect to the proton mass density. In the final expressions in (A14) and (A15) the ratios $\rho_{p} / \rho_{j}$ are to be interpreted as the ratios of the background mass densities. These final expressions are correct apart from a factor of sign $\left(q_{j}\right)$ which should multiply that in (A15)

(b) The middle expression in (A8) should be the imaginary part of the expression given. The final expression in terms of $\alpha_{\xi}$ is correct

(c) The specification "out of (in)" in the last paragraph on p5995 should be transposed to read "in (out of)"

mode is. Given a background plasma state, a wave mode is a normal mode or eigenstate of the system. The eigenvalue is usually taken to be the (complex) wave frequency $\omega$, with the (real) wave vector $\vec{k}$ prescribed. The associated eigenvector is the set of fluctuating fields $(\delta \vec{E}, \delta \vec{B})$ and plasma parameters $(\delta \vec{V}, \delta P, \delta \rho$ in the case of MHD or $\delta f_{j}(\vec{v})$ for kinetic waves). Mode identification is the process of comparing the observed eigenvalue/eigenvector with the possible theoretical ones and finding the one which matches.

The practical implementation of mode identification is strewn with pitfalls and complications, including:

- There is usually a mixture, possibly phase-coherent, of modes and/or frequencies, rather than an isolated mode.
- Frequencies are often Doppler shifted by an unknown, or poorly known, amount.

- Wave vectors are difficult to determine from the one(or few-) point measurements available from spacecraft.

- The mode eigenstate can depend sensitively on the wave vector, plasma $\beta$, and temperature anisotropy, as well as on the contributions of multi-species or nonMaxwellian kinetic features.

- The temporal resolution and accuracy of some measurements, notably those related to particle populations and plasma moments, are often insufficient or marginal. Some important species or sub-population may not be measured at all. The result is that either or both the background state or fluctuations are not accurately known. 
- Wave amplitudes are often large (of order unity), implying nonlinear processes may be present and distort the eigenstate from its linear form, or give rise to intrinsically nonlinear eigenstates.

- Similarly, inhomogeneities can distort the wave propagation or eigenstate in ways which have largely been unexplored.

- As we have seen, the theoretical wave properties depend on the framework in which the theory is performed. There is a trade-off between the simplicity/dubious applicability of MHD or fluid treatments and the complexity associated with the infinite degrees of freedom of kinetic theory.

Fortunately, there are only a few modes in the frequency range of consideration here. Thus, it is only necessary to establish a few key pieces of information to distinguish amongst these modes, as opposed to testing the complete eigenvector. Initially, only a few quantities were used in this process. These included the degree and handedness of the polarization of the magnetic field, the relative power in parallel versus transverse magnetic field fluctuations, in density fluctuations, etc. An ever-growing set of non-dimensional quantities, including "transport ratios" (Gary and Winske, 1992), polarizations, and other ratios has been devised. An extensive list is shown in Table 1. These ratios encapsulate distinctive aspects of the mode eigenvector, reducing the detail demanded from the observations, although many of the pitfalls itemized already remain problematical. Of particular concern is the sensitivity of some kinetic instabilities to background parameters, and to the dilution/contamination arising from a mixture of modes and/or frequencies. In general, ratios used for mode identification should be independent of reference frame, to avoid problems associated with Doppler shift or normalization to a parameter which is not fixed from one episode to the next (this view is not universally shared, however, as evidenced in the Doppler ratio of Song et al., 1994b). Table 1 shows a preponderance of ratios based entirely on the magnetic field. This emphasis has more to do with history and the relative completeness/higher sampling frequency of the field data by comparison with the plasma data than with the intrinsic capability of mode identification based on the magnetic field alone. These remarks are particularly relevant to the high $\beta$ conditions found in the magnetosheath, where the plasma dominates the field dynamics.

In Table 1 the subscript " $j$ " refers to species $p, e, \ldots$ for protons, electrons, etc., with $n, \vec{V}$ and $P$ corresponding to the species' density, bulk velocity, and thermal pressure respectively. Subscripts "o" denote background quantities. The coordinate system used is that of Krauss-Varban et al., (1994) in which $\vec{B}_{\mathrm{o}}=B_{\mathrm{o}} \hat{z}$ and the wave-vector $\vec{k}$ lies in the $\hat{x}-\hat{z}$ plane. The unit vector $\vec{\xi}$ lies in this plane and is perpendicular to $\vec{k}$ so that $\hat{\xi}, \hat{y}, \hat{k}$ form a right-handed system. The $\hat{y}$ direction is sometimes referred to as the noncoplanar direction. Transport ratios are defined in terms of the ratio of (cross)-correlation functions of fluctuating quantities. Under the assumption that the state is turbulent, stationary and homogeneous, such correlation functions can be Fourier analyzed and related to the complex Fourier amplitudes of the fluctuations. The oper- ator $\langle\delta a \delta b\rangle_{\vec{k} \omega}$ denotes such a Fourier transform of the cross-correlations between fluctuations in $a$ and $b$ (Gary and Winske, 1992).

There are two ways in which to apply the ratio approach to mode identification. The more traditional approach, stylized to the extreme in Fig. 1 of Song et al. (1994b), builds a decision tree in which a single ratio is used to eliminate some possibilities and choose which branch to follow further. Quantities such as a measure of the compressibility of the mode, phase between density and field fluctuations, transverse/non-coplanar field component and handedness of polarization are often particularly useful, although the last of these requires knowledge of the wave vector and phase velocity relative to the flow velocity. Application of such a tree structure, either algorithmically or case by case, is quite natural. The obvious danger of the tree approach is that an incorrect choice at a single node can be fatal. Such fatal choices can be induced by measurement errors, noise, or contamination of the eigenstate by the presence of more than one mode, nonlinearity, etc. Moreover, some information, which might be at odds with the final identification, may not be used if it only appears along some branches.

The alternative extreme applies a set of ratios, carefully chosen and weighted, together to measure the "distance" between an observed fluctuation and various theoretical ones in an $\mathrm{N}$-dimensional ratio space, as exemplified recently by Denton et al. (1995). This approach is also quite natural, with the benefit of never relying on any single ratio (and hence any single aspect of the observation) to eliminate possibilities. The problem lies in choosing a set of transport ratios from the list shown in Table 1 plus others yet to be invoked and in deciding how close is close enough to make a positive identification. The selected set should not bias the distance measure by repeated addition of essentially the same information. Worse still, the distance measure can allow positive identification even when one of the ratios on its own would exclude it, such as accepting an observation as being a parallel propagating Alfvén/ion-cyclotron mode when in fact it is right hand polarized. The method tries to cope with reality by establishing that the observed fluctuations are at least closer to this mode than that one, and thus leaves the door open for further scrutiny.

In practice, both extremes should be applied with caution, and both will cope adequately most of the time or fail simultaneously due to deficiencies in the data or applicability of the theory. Both these approaches rely on a comparison of the observations with expected values as derived from a particular theoretical framework. It should also be possible to classify the observations on their own, i.e., looking for clumps of observed events in some Ndimensional parameter space without an a priori attempt to pin down a particular theoretical mode. The average field spectra shown in Fig. 1 are an example of such an approach at a qualitative level and relying exclusively on field data.

Other identification schemes have also been developed. For example, Belmont and co-workers (Belmont and Mazelle, 1992; Belmont et al., 1992) introduce polytropic indices derived from kinetic wave properties into a set of 
fluid-like equations in an attempt to address the identification of compressive, ULF fluctuations via observations of fluid parameters. This also sheds light on the connection between MHD and kinetic solutions of the wave dispersion problem.

\section{Fast magnetosonic/whistler waves}

To date, no systematic study or search has reported low frequency fast modes in the magnetosheath. Even at large $\beta$ the fast/magnetosonic mode of kinetic theory (as defined by Krauss-Varban et al., 1994) is only lightly to moderately damped away from the cyclotron resonances and becomes the whistler mode at higher wave numbers. Fast magnetosonic waves can be driven unstable by fast ion beams, but these are not common in the magnetosheath. Whistler waves have been reported (Tsurutani et al., 1982) occurring in regions of low magnetic field strength, where they are referred to as "lion roars". Lion roars are believed to be excited by an electron anisotropy driven instability (Smith and Tsurutani, 1976).

\section{Alfvén/ion-cyclotron waves}

Alfvén/ion-cyclotron waves are driven by an ion temperature anisotropy $T_{\perp} / T_{\|}>1$, as is commonly found in the magnetosheath. The instability is strongest for parallely propagating modes $\left(\theta_{k B_{\mathrm{o}}} \sim 0^{\circ}\right)$ and is weakly dependent on the ion $\beta$, as shown in Fig. 3. The mode is left-hand polarized at parallel propagation in the plasma rest frame, being cyclotron resonant with the protons. Maximum growth corresponds to wave numbers $k \simeq 0.5-1.0 \omega_{p p} / c$, where $\omega_{p p}$ is the proton plasma frequency, as shown in

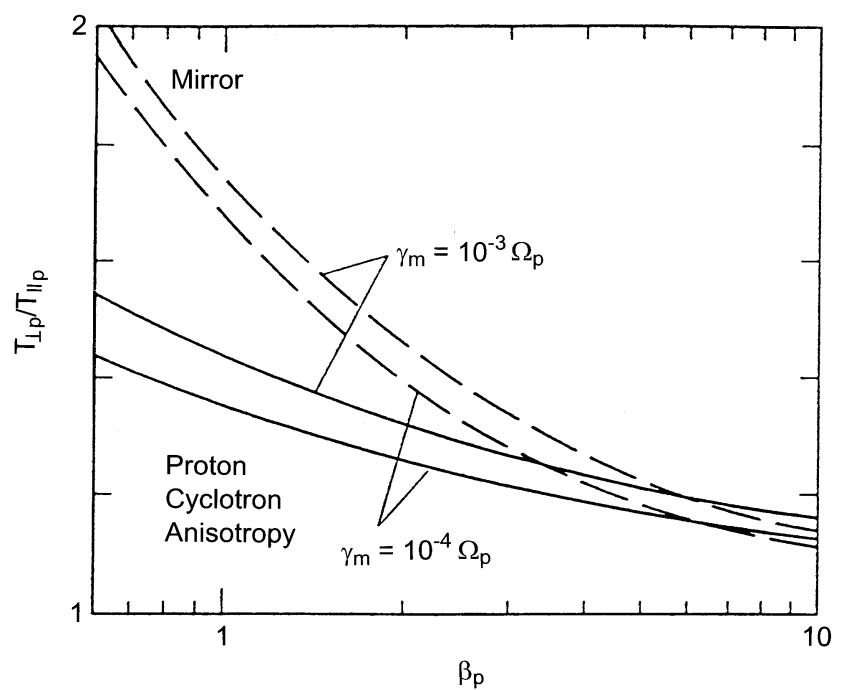

Fig. 3. Contours of constant maximum growth rate $\gamma_{m}$ in the $\beta$ anisotropy plane for the Alfvén/ion (proton)-cyclotron (solid curves) and mirror (dashed) instabilities. The Alfvén/ion-cyclotron instability is more weakly dependent on $\beta_{p}$ and requires a smaller $T_{\perp} / T_{\|}$at $\beta_{p} \sim 1$ for significant growth, (Fig. 2 of Gary et al., 1993b)
Fig. 4. The bottom panel of Fig. 4 also shows that the addition of alpha particles reduces the growth rate (Gary et al., 1993a; Gary et al., 1993b; Gary et al., 1994b) and introduces a second range of growth at lower frequencies and wave numbers which can be associated with the alpha particles. At high enough concentrations of cold alpha particles or proton anisotropies $\left(T_{\perp p} / T_{\| p}>4\right)$ a gap in frequency appears at $\Omega_{c \alpha}$ (Gary et al., 1993b). For low concentrations $(<1 \%)$ of alpha particles, an additional, marginally stable mode, sometimes termed the alpha cutoff mode, is found at very long wavelengths for frequencies just above the alpha particle cyclotron frequency (Lacombe et al., 1995).

An interesting example of a cyclotron mode is shown in Fig. 5. The polarization, field-aligned wave vector and expected growth based on $T_{\perp} / T_{\|}$are all consistent with an Alfvén/ion-cyclotron mode. The dual-spacecraft observations and good determination of $\pm \vec{k}$ via the minimum variance technique greatly assisted the analysis in this case. However, the Alfvén/ion-cyclotron mode has an electron parallel compressibility, $C_{\| e}$, significantly greater than one (see, for example, Fig. 11 of Krauss-Varban et al., 1994), while that for the wave shown in Fig. 5 is only $\sim 1$. Moreover, the phase of the electron density-field ratio, $C_{p e}$, i.e., the phase between the parallel magnetic field fluctuations and the density, is expected to be $\sim-70^{\circ}$ while the observed value is $\sim-15^{\circ}$. Although there are observations of waves more closely matching expectations (Lacombe et al., 1995), this example serves to highlight the

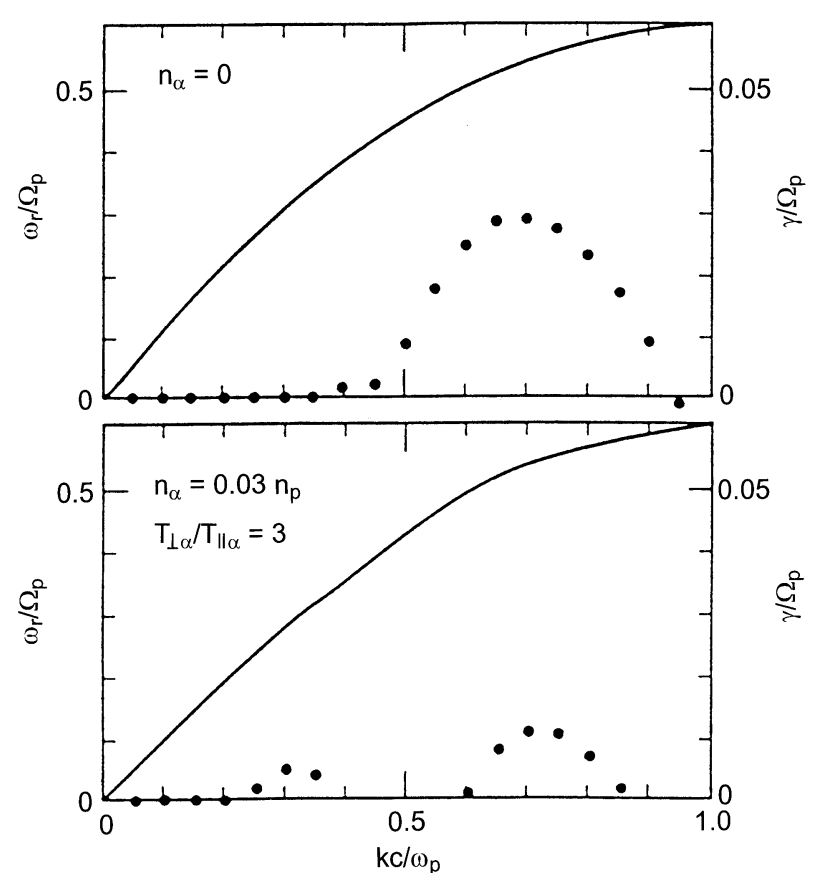

Fig. 4. Real frequencies (solid) and growth rates (dots) for the parallel-propagating Alfvén/ion-cyclotron instability in the case of no alpha particles (top) and a 3\% alpha particle concentration (bottom). Proton parameters are $\beta_{p}=0.25$ and $T_{\perp p} / T_{\| p}=2.5$. The alpha particles have $T_{\| \alpha}=T_{\| p}$ and $T_{\perp \alpha} / T_{\| \alpha}=3$. The addition of anisotropic alpha particles introduces a second region of instability at $\omega_{r} \lesssim \Omega_{c \alpha}$ which can be identified as an Alfvén/ion (alpha)-cyclotron mode, (from Fig. 3 of Gary et al., 1993b) 


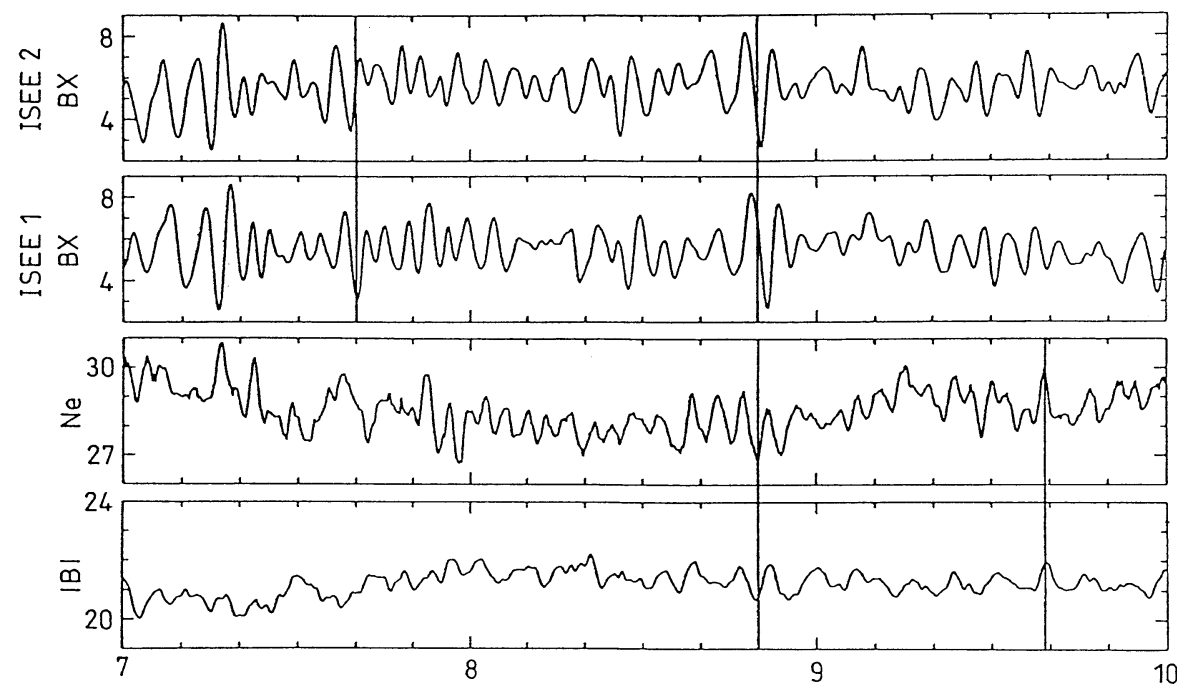

Fig. 5. Dual spacecraft magnetic waveforms (top two panels), electron density (third panel) and field magnitude (bottom) showing a possible Alfvén/ion-cyclotron wave. However, the magnitude and phase of the electron density-field ratio $C_{p e}$ do not match that of the theory, leading the original authors to suggest that this is an example of a coherent, mixed-mode proton/alpha-cutoff cyclotron mode. (Fig. 3 of Lacombe et al., 1995) kinds of difficulties faced in attempting to match observed fluctuations with particular wave modes/instabilities.

Recent attempts to illustrate the role of the Alfvén/ioncyclotron modes in the magnetosheath have stressed the mode's ability to pitch-angle scatter particles, as revealed by one- and two-dimensional hybrid simulations (McKean et al., 1992b; Gary and Winske, 1993; Gary et al., 1993c; McKean et al., 1994). This scattering thereby results in a more-thermalized equilibrium state (Anderson et al., 1994; Denton et al., 1994b; Gary and Lee, 1994; Gary et al., 1993a; Gary et al., 1994a; Gary et al., 1994b; Gary et al., 1994c). Moreover, this work established a significant correlation between the proton temperature anisotropy and the proton $\beta$ in the magnetosheath, and one which has the same slope as that required to keep the Alfvén/ion-cyclotron mode near to marginal stability. This paradigm suggests that these waves are being driven within the magnetosheath and in turn react back on the particle distributions to reduce the mode growth rate from large values to that corresponding to further macroscopic pumping rates. Artificially driven simulations (Gary et al., 1994c; Denton et al., 1993) have been used to support this paradigm, although they do not completely represent the pumping processes in the magnetosheath. The Alfvén/ ion-cyclotron mode should be particularly effective at low $\beta$. At higher $\beta$ 's, the mirror mode should grow more readily (Gary, 1992; Gary et al., 1993b) (see Fig. 3), especially as the inclusion of alpha particles suppresses the Alfvén/ion-cyclotron mode in the regime of larger anisotropies.

\section{Mirror modes}

The mirror mode is unusual in that it has zero frequency in the plasma rest frame. The density and magnetic field fluctuations are in strict anti-phase (i.e., $R_{\| n}=-1$, which is also reflected in the phase of the density-field ratio $C_{p j}$ ). Figure 6 shows one example of a mirror mode. Other examples have been reported, including observations within the Earth's magnetosheath (Tsurutani et al., 1982;

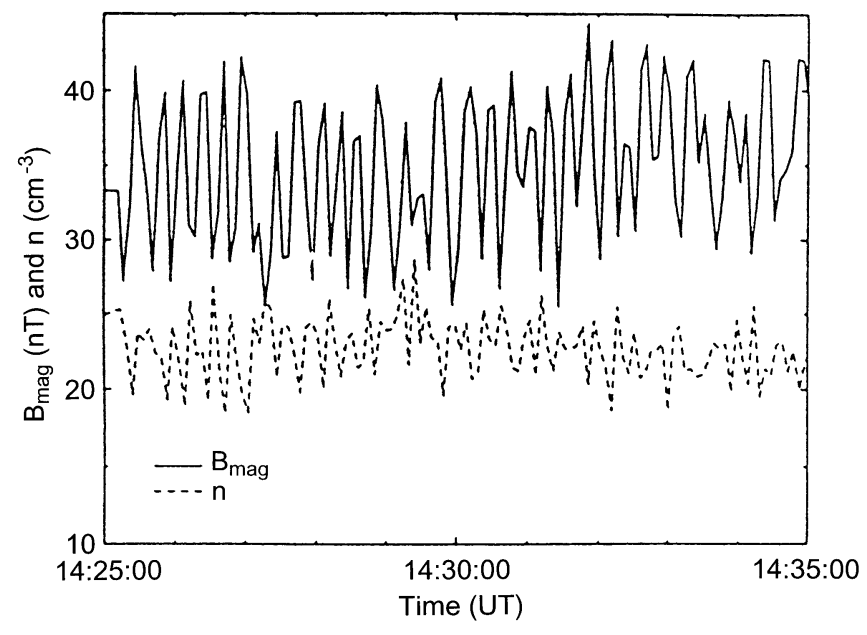

Fig. 6. Magnetic field (solid) and ion density (dashed) measurements showing a nearly sinusoidal mirror mode. Note the strong anticorrelation between the field and density. (Fig. 1 of Leckband et al., 1995).

Tsurutani et al., 1984; Hubert et al., 1989; Lacombe et al., 1992; Fazakerley and Southwood, 1994a; Fazakerley et al., 1995), the Jovian environment (Balogh et al., 1992; Phillips et al., 1993), the Saturnian environment (Violante et al., 1995a, b), the Uranian environment (Russell et al., 1989), cometary environs (Russell et al., 1987; Mazelle et al., 1991; Glassmeier et al., 1993), and interplanetary space (Tsurutani et al., 1992; Winterhalter et al., 1994). The mirror mode is unstable in kinetic theory for temperature anisotropies satisfying

$\frac{T_{\perp}}{T_{\|}}>1+\frac{1}{\beta_{\perp}}$

(Hasegawa, 1969) which is similar to that found in mixed kinetic-fluid treatments, e.g. (Southwood and Kivelson, 1993), and disagrees by a factor of six with the result in the CGL approximation given in Eq. (1). The threshold in Eq. (2) confirms that the mirror mode is favoured by large $\beta$. 
As this threshold is reached, the first unstable mode has a wave vector $\vec{k}$ which is nearly perpendicular to $\vec{B}_{\text {o }}$. As the anisotropy is increased, however, the most unstable mode shifts to more oblique $\vec{k}$ 's, reaching $\theta_{k B_{\circ}} \sim 60^{\circ}$ for $T_{\perp} / T_{\|} \geqslant 2$ (Pantellini and Schwartz, 1995; Krauss-Varban et al., 1994). Warm/hot electrons modify the instability threshold to some extent, and decrease the growth rate (Pantellini and Schwartz, 1995).

The physics of the mirror instability has received renewed interest in recent years (Southwood and Kivelson, 1993) following a long period of neglect (Rose, 1965). The fluid perspective starts, paradoxically, from the adiabatic particle motion in a non-uniform magnetic field and Liouville's Theorem. The second moment of the deviations of an initially anisotropic bi-Maxwellian distribution yields the following relationship between the particle and the magnetic field pressures:

$\delta P_{\perp}=\beta_{\perp}\left(1-\frac{T_{\perp}}{T_{\|}}\right) \delta\left(B^{2} / 2 \mu_{\mathrm{o}}\right)$

(Note that this is not consistent with CGL theory.) Thus, if $T_{\perp}>T_{\|}$the variations in particle and magnetic field pressures transverse to the background field are in antiphase. Moreover, at large $\beta_{\perp}$ the deviation in particle pressure is too large to be compensated by that of the magnetic field, and instability results.

Viewed from the kinetic perspective, the previous argument applies to non-resonant particles, i.e., those which "circulate" freely through the mode crests and troughs. Resonant particles remain near a fixed phase point, i.e., they satisfy $k_{\|} v_{\|} \simeq \omega_{r}$. Since $\omega_{r}=0$, resonant particles in the case of the mirror instability are those with parallel speeds near zero. These particles see the growing wave field and are betatron accelerated (decelerated) in regions of field maximum (minimum), thus the change in their perpendicular energy is in-phase with the magnetic field, and they act to bring about near pressure equilibrium. A curious twist is then that the fewer the number of resonant particles, the less they are able to compensate and hence the faster the mode will grow (Southwood and Kivelson, 1993). Figure 7 shows the resulting distribution function expected in the mirror mode. Pantellini et al. (1994) have developed methods for extracting the distribution functions expected for modes based on kinetic theory, while Leckband et al. (1995) have made tentative identification of the features predicted for the mirror modes (e.g., Fig. 8 of Pantellini and Schwartz, 1995) from satellite data.

Figure 8 shows the real frequency and growth rates of the low-frequency modes based on kinetic theory. This figure reveals the competitive nature of the mirror mode at large anisotropy and the simultaneous existence of a heavily damped slow mode. Careful choice of transport ratios (see Fig. 8b of Krauss-Varban et al., 1994) should facilitate identification of these different modes, as attempted by Denton et al. (1995). Figure 9 shows that the electron compressibility $C_{e}$ of certain predominant classes of compressive magnetosheath waves indicate the presence of mirror modes, since they have compressibilities less than unity (the slow mode has compressibilities $>1$, Gary, 1992) and show density and magnetic field fluctuations in
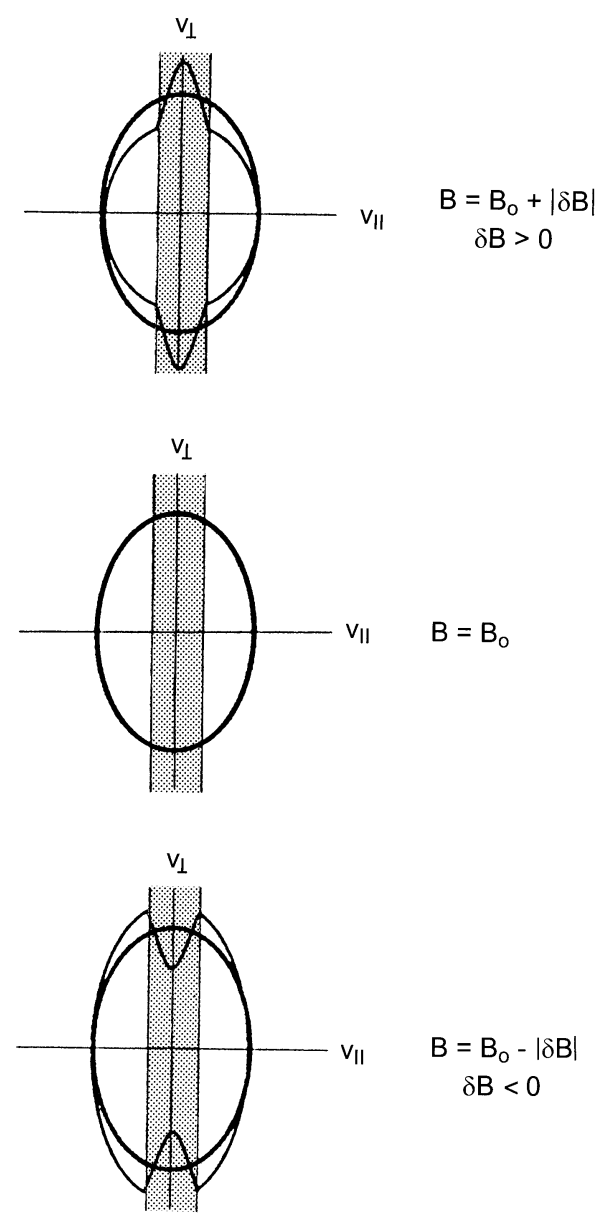

range of $v_{\|}<\gamma / k_{\|}$

Fig. 7. Contours of constant phase space density in the peak (top), node (centre) and trough of magnetic field strength of a mirror mode. The solid curves correspond to the initial state. The behaviour of the resonant particles near $v_{\|}=0$ is due to the secular betatron acceleration/deceleration and is opposite to the remaining circulating particle behaviour. The circulating particles respond adiabatically and the deformation of the contours in these regions is a simple consequence of Liouville's Theorem for inhomogeneous quasi-stationary fields. (Fig. 2 of Southwood and Kivelson, 1993)

anti-phase. (Note that the caption to Fig. 4 of Anderson et al., 1994 refers to $C_{e}$ as the parallel compressibility and that the definition of $C_{e}$ given in their text is not that used in the cited work; see Table 1 and Gary, 1992.)

Hybrid particle simulations (e.g., McKean et al., 1992a; McKean et al., 1993) reveal that the mirror fluctuations persist long after saturation due to their inability to effectively scatter the particles. Under extreme conditions, nonlinear mirror modes give rise to longer wavelength monoliths in $B$. Signatures of particle trapping between such monoliths are found in the data (Leckband et al., 1995) and hybrid simulations (Pantellini et al., 1995, also private communication). Recently, Kivelson and Southwood (1995) have explored qualitatively the role of such trapping in establishing the marginally stable state of the mirror mode. 

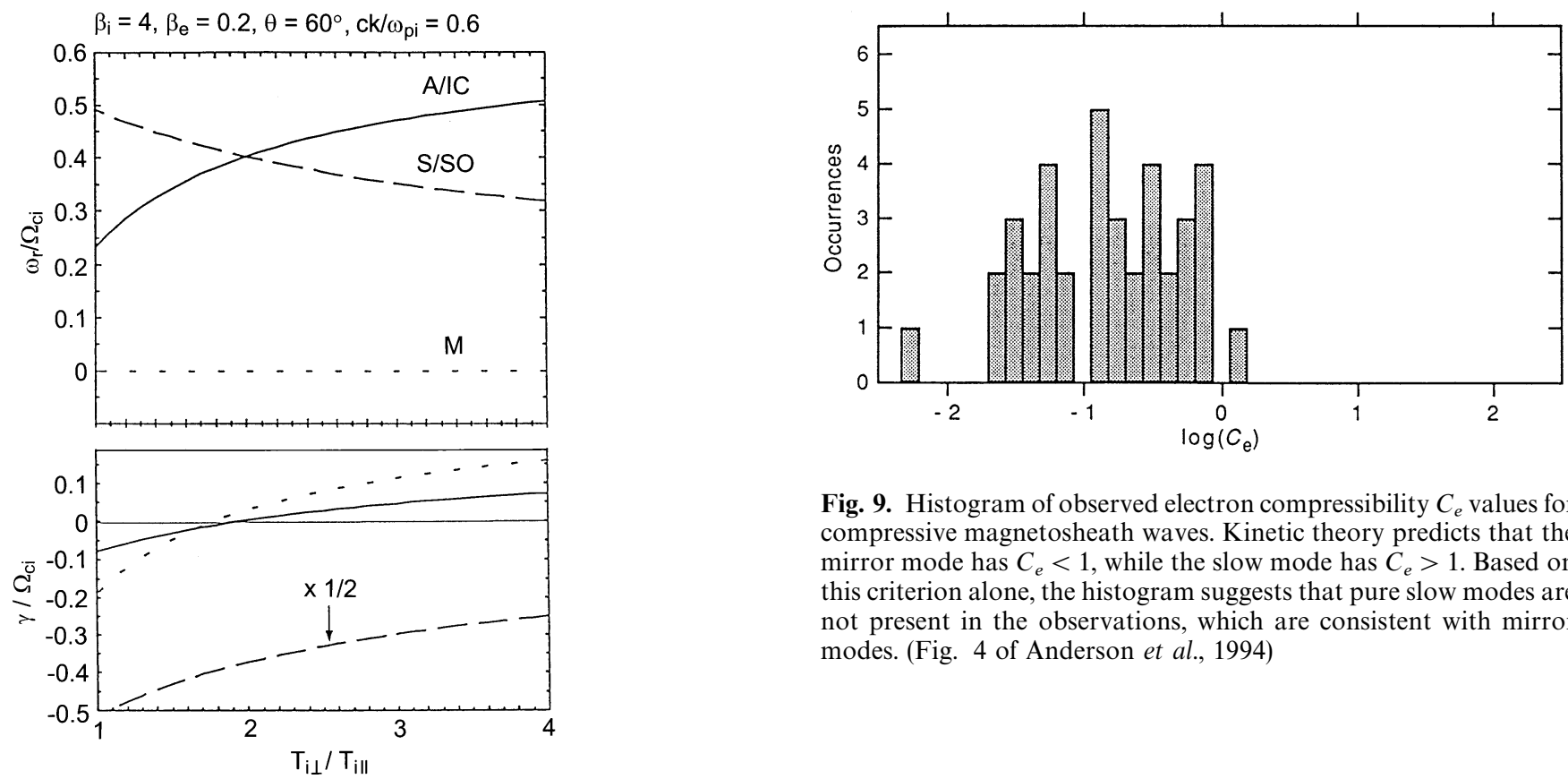

Fig. 9. Histogram of observed electron compressibility $C_{e}$ values for compressive magnetosheath waves. Kinetic theory predicts that the mirror mode has $C_{e}<1$, while the slow mode has $C_{e}>1$. Based on this criterion alone, the histogram suggests that pure slow modes are not present in the observations, which are consistent with mirror modes. (Fig. 4 of Anderson et al., 1994)

Fig. 8. Real frequencies (top) and growth rates (bottom) of the Alfvén/ion-cyclotron, slow/sound, and mirror modes as a function of ion temperature anisotropy from kinetic theory. The $A / I C$ and $M$ modes show roughly comparable growth rates over a range of anisotropies, with the mirror instability stronger by a factor $\sim 2$. Kinetic theory reveals the simultaneous presence of the slow mode, which is heavily damped. (Fig. 8a of Krauss-Varban et al., 1994)

Fig. 10. Power spectra of the ion thermal pressure (top) and magnetic pressure (second panel) of fluctuations within the slow-mode transition near the sub-solar magnetopause. The coherence (third panel) and anti-phase (bottom) of the magnetic and thermal pressure fluctuations is consistent with either the slow mode or mirror mode. The rather low frequency of the feature at $\sim 0.008 \mathrm{~Hz}$ suggests that these waves are nearly standing against the magnetosheath flow. The authors conclude, therefore, that they cannot be pure mirror modes. (Fig. 5 of Song et al., 1992b)
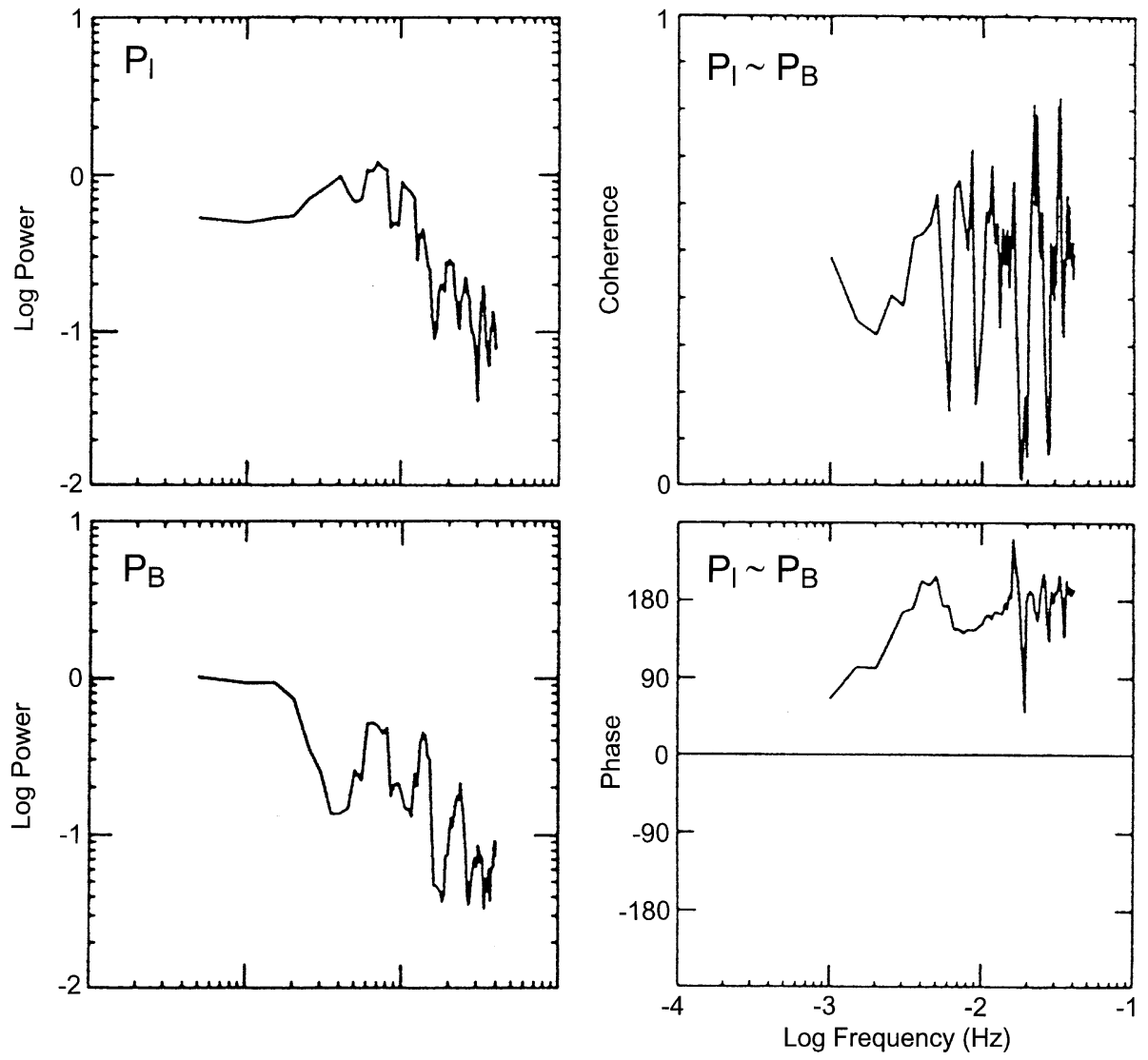


\section{Slow/sound waves}

Slow mode waves should be heavily damped under magnetosheath conditions (e.g., Fig. 8). Moreover, the slow mode is heavily guided by the background magnetic field direction, so that the near perpendicular configuration created by magnetic field draping near the sub-solar region of the magnetopause is unfavourable for its propagation. However, along the stagnation line the bulk flow clearly drops below the slow mode speed. A standing slow mode front is expected and observed (Song et al., 1992a; Song et al., 1995; Southwood and Kivelson, 1992; Yan and Lee, 1994). Thus, the magnetopause is clearly capable of generating a slow mode disturbance of some kind, although a single standing front (shock?) does not by itself imply that small amplitude waves will also propagate.

Song et al. (1992b) do report low-frequency wavetrains with thermal and magnetic pressure variations in antiphase and which apparently stand in the flow, i.e. they have a finite propagation speed, based on dual spacecraft timings. Their example is shown in Fig. 10. On the basis of linear theory, only the slow mode could possibly account for these observations (although the value of the flow velocity used is not given). Perhaps, therefore, the arguments about the slow mode being heavily damped do not reveal the whole story in the structured, inhomogeneous environment near the magnetopause. Alternatively, perhaps the inhomogeneous environment alters the properties of, say, the non-propagating mirror mode or couples the two modes (see later).

One of the principal difficulties is the identification of the $\vec{k}$-vector, as the conversion from spacecraft timings to propagation speeds depends critically on the direction of $\vec{k}$. Sometimes (e.g., Song et al., 1992b), different methods give widely different answers. An alternative approach is to perform the process in reverse, using the spacecraft timings to constrain possible orientations of $\vec{k}$ based on the phase speeds of likely wave modes (Gleaves and Southwood, 1990, 1991). This method provides a complementary approach, but relies heavily on the plasma parameters and theoretical framework for the wave modes. It is also possible to jump to the erroneous conclusion (Gleaves and Southwood, 1991) that if the downstream spacecraft sees the fluctuation first, the fluctuation cannot be a convected structure/mirror wave, as this conclusion, while natural, depends again on the precise orientation of $\vec{k}$.

Computer simulations provide one means of overcoming the difficulties inherent in single-point (or even fewpoint) satellite measurements by supplying complete spatial and temporal information within the computational domain. Omidi and Winske (1995) used a one-dimensional hybrid simulation to model the entire region from the solar wind to the magnetopause (or more correctly, a "magnetic boundary" since their simulation had a finite normal component of $\vec{B}$ and mass flux). The results are shown in Fig. 11. The fast shock ("FS") is the left-most feature and propagates to the left. To its right are a set of mirror modes which convect and pass through a more slowly propagating rotational discontinuity ("RD"). The fluctuations to the right of the RD are still mirror modes, which were amplified on their passage through the RD. Immediately to the left of the "magnetic boundary", however, are a few cycles of fluctuations which appear to be phase standing relative to the "magnetic boundary". Since there is a net mass flux through the boundary, these phase standing waves must have a finite propagation speed in the plasma rest frame, and hence cannot be just mirror modes in the classical sense. Wave analysis (e.g., Fig. 10 of Omidi and Winske, 1995) confirms that the mode has a finite real frequency and a compressibility $C_{p} \sim 0.5$ which is near but not identical to that for a mirror mode is the local conditions $(\sim 0.1)$. On the other hand, the mode frequency and compressibility are respectively one and two orders of magnitude below that of a pure slow mode of the same $k$. Omidi and Winske (1995) refer to these modes as "MIrror And slOW" or MIAOW waves, although as inferred above they are much "closer" to mirror waves than to the slow mode. Thus, the vicinity of the magnetic boundary would appear to modify the characteristics of the mirror mode to provide it with a finite phase velocity or, as Omidi and Winske (1995) phrase it, to allow some slippage of the plasma with respect to the field. These effects may be due to a variety of processes, including nonlinearity and mode-coupling in the inhomogeneous background state.

These simulations bear a remarkable resemblance to the observations of the slow-mode transition by Song et al. (1992a). The question of whether there are any observations of modes matching more closely slow-mode wave trains remains an open one.

\section{Discussion}

We have seen that the magnetosheath is typically a high- $\beta$, anisotropic environment. Particle reflection at the bow shock and the ion foreshock provide upstream sources of turbulence and free energy to drive local instabilities. Field-line draping and compression at the magnetopause, along with the magnetopause itself, provide sources of free energy which are able to influence the local plasma and turbulence in the magnetosheath. The result is a turbulent magnetosheath with significant power over a wide range of the low-frequency spectrum. The high- $\beta$ anisotropic conditions require a kinetic description; fluid theories of various kinds provide some insight into the possible wave mode properties, but cannot describe the plasma instabilities which arise and give misleading wave characteristics on some occasions.

Two wave modes seem to dominate the magnetosheath, and grow there due to the ion $T_{\perp}>T_{\|}$anisotropy which pervades throughout. The Alfvén/ion-cyclotron mode grows readily under modest $\beta$ conditions, and is thus found behind the weaker quasi-perpendicular bow shocks and in the plasma depletion layer. At higher $\beta$, e.g., behind strong quasi-perpendicular bow shocks and in the middle magnetosheath, the zero-frequency compressive mirror mode dominates the power spectra at low frequencies. A modified version of the mirror mode, with finite frequency and propagation speed, appears to exist downstream of the slow-mode transition close to the subsolar magnetospause. 

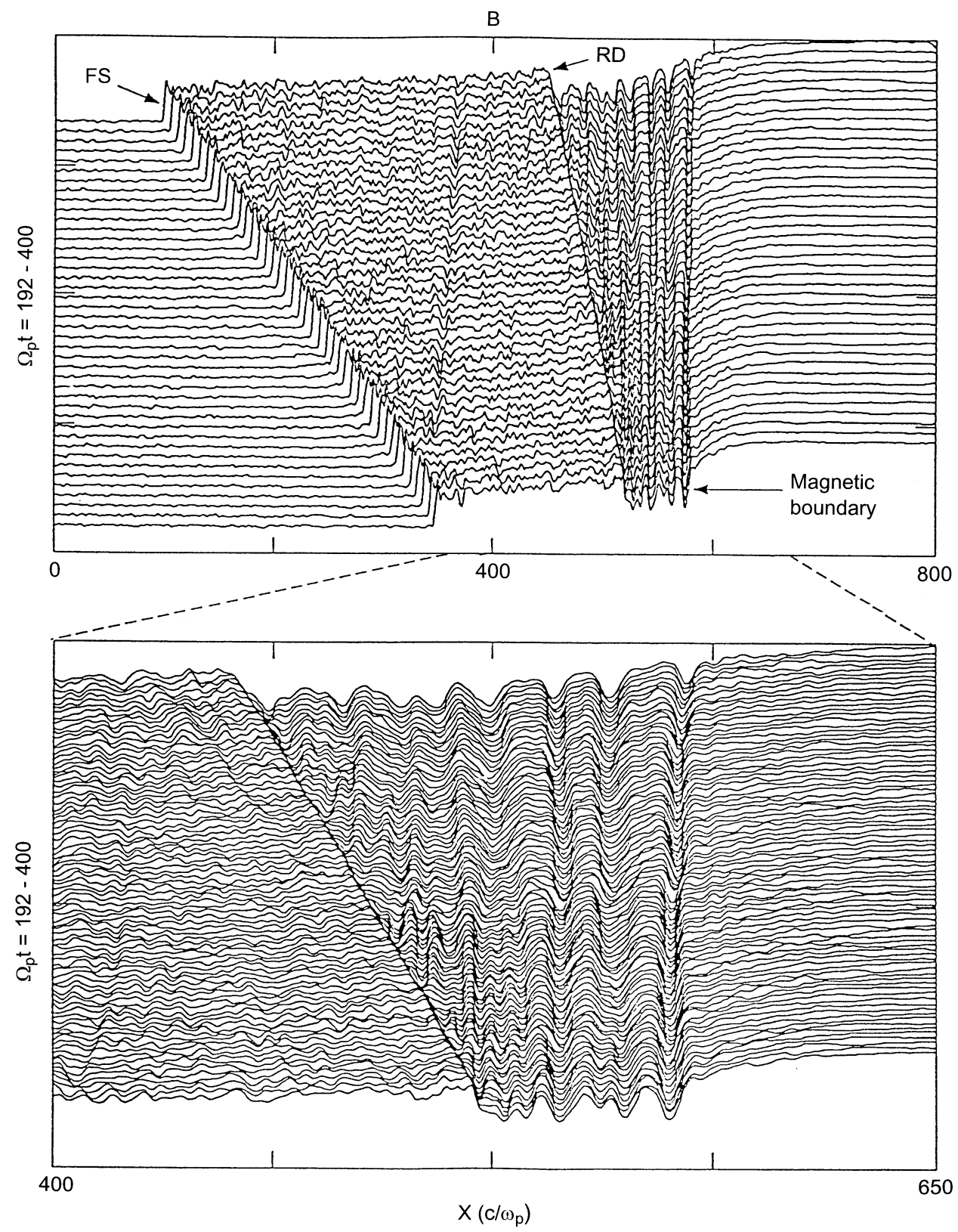

Fig. 11. One dimensional hybrid simulations of a flow incident on a magnetic boundary with non-zero normal field (and mass flux). The figure shows the magnetic field profile within the box stacked above one another for various successive times. A fast shock $(F S)$ propagates leftward with convecting mirror waves in the downstream region to its right. These mirror modes become amplified by the rotational discontinuity $(R D)$ but then appear to stand against the flow just upstream of the magnetic boundary, implying a non-zero phase speed there. (Fig. 9 of Omidi and Winske, 1995)

These generalities mask a number of complications and difficulties associated with making a positive identification of a particular wave mode. Pure modes are rarely seen; instead a mixture of modes over a range of frequencies complicate the identification process. Such a mixture may be partially coherent, thereby mixing in an inseparable manner the mode characteristics. Mode properties and nomenclature rely on linear theory in a homogeneous plasma, often based on fluid/MHD theories of some kind. The real magnetosheath is a highly structured, inhomogeneous medium with strong kinetic aspects. Many of the parameters or "transport ratios" used as an aid to identification, and the wave dispersion/growth characteristics, can all be sensitive to plasma details (composition, anisotropy, wave-vector orientations, etc.) which are difficult to measure with precision.

There are several areas where improvement is required and achievable. Perhaps the most important problem is the determination of the wave vector $\vec{k}$. While this is possible under some circumstances, often traditional single-spacecraft techniques do not yield reliable estimates. Knowledge of $\vec{k}$ would enable the Doppler shift due to the magnetosheath bulk flow to be removed, thus revealing the waves' most basic quantity: the wave dispersion. The application of more modern techniques (e.g., Dudok de Wit et al., 1995) and the multipoint measurements of Cluster should greatly advance this area. Several multipoint analysis techniques have been developed recently (Glassmeier et al., 1995; Neubauer and Glassmeier, 1990; Pinçon and Lefeuvre, 1991; Pinçon, 1993; vom Stein et al., 1993).

Another area where observations could be readily improved is in revealing the kinetic response of the particle distribution functions (e.g., Leckband et al., 1995; Pantellini et al., 1994; Pantellini et al., 1995), rather than relying on quantities based on moments of the distribution 
function. Features such as resonant particles again relate to fundamental wave/instability properties.

Finally, better theory/simulation dealing with the inhomogeneous, nonlinear, mixed-mode regimes common to the magnetosheath would be welcomed. Such theoretical efforts not only provide spatial and temporal detail not possible in space, but also enable spacecraft techniques to be tested and refined.

Acknowledgements. This work grew out of a review presented at the XXI IUGG Assembly in Boulder, Colorado, July 1995. We are grateful for the encouragement of several attendees to write this review. Xochitl Blanco-Cano and Dietmar Krauss-Varban provided helpful comments. This work was supported by the Particle Physics and Astronomy Research Council of the UK via Grants GR/J36440 and GR/J88388.

Topical Editor K.-H. Glaßmeier thanks C. Mazelle and R. E. Denton for their help in evaluating this paper.

\section{References}

Abraham-Shrauner, B., Propagation of hydromagnetic waves through an anisotropic plasma, J. Plasma Phys., 1, 361-378, 1967.

Anderson, B. J., and S. A. Fuselier, Magnetic pulsations from 0.1 to $4.0 \mathrm{~Hz}$ and associated plasma properties in the Earth's subsolar magnetosheath and plasma depletion layer, J. Geophys. Res., 98, 1461-1479, 1993.

Anderson, B. J., S. A. Fuselier, S. P. Gary, and R. E. Denton, Magnetic spectral signatures in the Earth's magnetosheath and plasma depletion layer, J. Geophys. Res., 99, 5877-5891, 1994.

Balogh, A., M. K. Dougherty, R. J. Forsyth, D. J. Southwood, E. J. Smith, B. T. Tsurutani, N. Murphy, and M. E. Burton, Magnetic field observations in the vicinity of Jupiter during the Ulysses flyby, Science, 257, 1515, 1992.

Belmont, G., and C. Mazelle, Polytropic indexes in collisionless plasmas - theory and measurements, J. Geophys. Res., 97, 8327-8336, 1992.

Belmont, G., D. Hubert, C. Lacombe, and F. Pantellini, Mirror mode and other compressive ULF modes, Proceedings of the 26th ESLAB Symposium, Killarney, Ireland, 16-19 June 1992, ESA SP-346, 263-267, 1992.

Chew, G. F., M. L. Goldberger, and F. E. Low, The Boltzmann equation and the one-fluid hydromagnetic equations in the absence of particle collisions, Proc. R. Soc., A236, 112, 1956.

Crooker, N. U., T. E. Eastman, and G. S. Stiles, Observations of plasma depletion in the magnetosheath at the magnetopause, J. Geophys. Res., 84, 869-874, 1979.

Denton, R. E., M. K. Hudson, S. A. Fuselier, and B. J. Anderson, Electromagnetic ion-cyclotron waves in the plasma depletion layer, J. Geophys. Res., 98, 13477-13490, 1993.

Denton, R. E., B. J. Anderson, S. P. Gary, and S. A. Fuselier, Bounded anisotropy fluid model for ion temperatures, J. Geophys. Res., 99, 11225-11241, 1994a.

Denton, R. E., S. P. Gary, B. J. Anderson, S. A. Fuselier, and M. K. Hudson, Low-frequency magnetic fluctuation spectra in the magnetosheath and plasma depletion layer, J. Geophys. Res., 99, 5893-5901, 1994b.

Denton, R. E., S. P. Gary, X. L. Li, B. J. Anderson, J. W. Labelle, and M. Lessard, Low-frequency fluctuations in the magnetosheath near the magnetopause, J. Geophys. Res., 100, 5665-5679, 1995.

Dudok de Wit, T., V. V. Krasnosel'skikh, S. D. Bale, M. W. Dunlop, H. Lühr, S. J. Schwartz, and L. J. C. Woolliscroft, Determination of dispersion relations in quasi-stationary plasma turbulence using dual satellite data, Geophys. Res. Lett., 22, 2653-2656, 1995.

Farrugia, C. J., R. P. Rijnbeek, M. A. Saunders, D. J. Southwood, D. J. Rodgers, M. F. Smith, C. P. Chaloner, D. S. Hall, P. J. Christiansen, and L. J. C. Woolliscroft, A multi-instrument study of flux-transfer event structure, J. Geophys. Res., 93, 14465, 1988.
Fazakerley, A. N., and D. J. Southwood, Mirror instability in the magnetosheath, Adv. Space Res., 14, 65-68, 1994a.

Fazakerley, A. N., and D. J. Southwood, Theory and observation of magnetosheath waves, in Solar Wind Sources of Magnetospheric Ultra-Low Frequency Waves, pp. 147-158, American Geophysical Union, 1994b.

Fazakerley, A. N., A. J. Coates, and M. W. Dunlop, AMPTE-UKS observations of ion velocity distributions associated with magnetosheath waves, Adv. Space Res., 15, 349, 1995.

Fuselier, S. A., B. J. Anderson, S. P. Gary, and R. E. Denton, Inverse correlations between the ion temperature anisotropy and plasma-beta in the Earth's quasi-parallel magnetosheath, J. Geophys. Res., 99, 14931-14936, 1994.

Gary, S. P., The mirror and ion-cyclotron anisotropy instabilities, J. Geophys. Res., 97, 8519-8529, 1992.

Gary, S. P., Theory of space plasma microinstabilities, Cambridge University Press, New York, 1993

Gary, S. P., and M. A. Lee, The ion-cyclotron anisotropy instability and the inverse correlation between proton anisotropy and proton-beta, J. Geophys. Res., 99, 11297-11301, 1994.

Gary, S. P., and D. Winske, Correlation-function ratios and the identification of space plasma instabilities, J. Geophys. Res., 97, 3103-3111, 1992.

Gary, S. P., and D. Winske, Simulations of ion-cyclotron anisotropy instabilities in the terrestrial magnetosheath, J. Geophys. Res., 98 9171-9179, 1993.

Gary, S. P., B. J. Anderson, R. E. Denton, S. A. Fuselier, M. E. McKean, and D. Winske, Ion anisotropies in the magnetosheath, Geophys. Res. Lett., 20, 1767-1770, 1993a.

Gary, S. P., S. A. Fuselier, and B. J. Anderson, Ion anisotropy instabilities in the magnetosheath, J. Geophys. Res., 98 1481-1488, 1993b.

Gary, S. P., M. E. McKean, and D. Winske, Ion-cyclotron anisotropy instabilities in the magnetosheath - theory and simulations, J. Geophys. Res., 98, 3963-3971, 1993c.

Gary, S. P., B. J. Anderson, R. E. Denton, S. A. Fuselier, and M. E. McKean, A limited closure relation for anisotropic plasmas from the Earth's magnetosheath, Phys. Plasmas, 1, 1676-1683, 1994a.

Gary, S. P., P. D. Convery, R. E. Denton, S. A. Fuselier, and B. J. Anderson, Proton and helium cyclotron anisotropy instability thresholds in the magnetosheath, J. Geophys. Res., 99, 5915-5921, 1994b.

Gary, S. P., M. E. McKean, D. Winske, B. J. Anderson, R. E. Denton, and S. A. Fuselier, The proton cyclotron instability and the anisotropy beta-inverse correlation, J. Geophys. Res., 99, 5903-5914, 1994c.

Glassmeier, K. H., U. Motschmann, C. Mazelle, F. M. Neubauer, K. Sauer, S. A. Fuselier, and M. H. Acuna, Mirror modes and fast magnetoacoustic waves near the magnetic pileup boundary of Comet-p Halley, J. Geophys. Res., 98, 20955-20964, 1993.

Glassmeier, K. H., U. Motschmann, and R. v. Stein, Mode recognition of MHD wave fields at incomplete dispersion measurements, Ann. Geophysicae, 13, 76-83, 1995.

Gleaves, D. G., and D. J. Southwood, Phase delays in transverse disturbances in the Earth's magnetosheath, Geophys. Res. Lett. 17, 2249-2252, 1990

Gleaves, D. G., and D. J. Southwood, Magnetohydrodynamic fluctuations in the Earth's magnetosheath at 1500 LT-ISEE-1 and ISEE-2, J. Geophys. Res., 96, 129-142, 1991.

Gosling, J. T., and A. E. Robson, Ion reflection, gyration, and dissipation at supercritical shocks, in Collisionless Shocks in the Heliosphere: Reviews of Current Research, pp. 141-152, American Geophysical Union, 1985.

Gosling, J. T., M. F. Thomsen, S. J. Bame, and C. T. Russell, Ion reflection and downstream thermalization at the quasi-parallel bow shock, J. Geophys. Res., 94, 10,027-10,037, 1989.

Greenstadt, E. W., C. T. Russell, V. Formisano, P. C. Hedgecock, F. L. Scarf, M. Neugebauer, and R. E. Holzer, Structure of a quasi-parallel, quasi-laminar bow shock, J. Geophys. Res., 82, 651, 1977.

Hasegawa, A., Drift mirror instability of the magnetosphere, Phys. Fluids, 7, 897-900, 1969. 
Hau, L. N., and B. U. O. Sonnerup, On slow-mode waves in an anisotropic plasma, Geophys. Res. Lett., 20, 1763-1766, 1993.

Hill, P., G. Paschmann, R. A. Treumann, W. Baumjohann, and H. Lühr, Plasma and magnetic-field behavior across the magnetosheath near local noon, J. Geophys. Res., 100, 9575-9583, 1995.

Hubert, D., Nature and origin of wave modes in the dayside Earth magnetosheath, Adv. Space Res., 14, 55-64, 1994.

Hubert, D., C. Perche, C. C. Harvey, C. Lacombe, and C. T. Russell, Observation of mirror waves downstream of a quasi-perpendicular shock, Geophys. Res. Lett., 16, 159-162, 1989.

Kivelson, M. G., and D. J. Southwood, Mirror instability II: the mechanism of non-linear saturation, J. Geophys. Res., in press, 1995.

Krauss-Varban, D., Bow shock and magnetosheath simulations: Wave transport and kinetic properties, in Solar Wind Sources of Magentospheric Ultra-low Frequency Waves, pp. 121-134, American Geophysical Union, 1994.

Krauss-Varban, D., N. Omidi, and K. B. Quest, Mode properties of low-frequency waves - kinetic-theory versus Hall-MHD, J. Geophys. Res., 99, 5987-6009, 1994.

Lacombe, C., and G. Belmont, Waves in the Earth's magnetosheath: observations and interpretations, Adv. Space Res., 15, (8/9)329-(8/9)340, 1995.

Lacombe, C., E. Kinzelin, C. C. Harvey, D. Hubert, A. Mangeney, J. Elaoufir, D. Burgess, and C. T. Russell, Nature of the turbulence observed by ISEE 1-2 during a quasi-perpendicular crossing of the Earth's bow shock, Ann. Geophysicae, 8, 489-502, 1990

Lacombe, C., F. G. E. Pantellini, D. Hubert, C. C. Harvey, A. Mangeney, G. Belmont, and C. T. Russell, Mirror and Alfvénic waves observed by ISEE 1-2 during crossings of the Earth's bow shock, Ann. Geophysicae, 10, 772-784, 1992.

Lacombe, C., G. Belmont, D. Hubert, C. C. Harvey, A. Mangeney, C. T. Russell, J. T. Gosling, and S. A. Fuselier, Density and magnetic-field fluctuations observed by ISEE-1-2 in the quiet magnetosheath, Ann. Geophysicae, 13, 343-357, 1995.

Leckband, J. A., D. Burgess, S. J. Schwartz, and F. G. E. Pantellini, Ion distributions associated with mirror waves in the Earth's magnetosheath, Adv. Space Res., 15, 345-348, 1995.

Mazelle, C., G. Belmont, K.-H. Glassmeier, D. Le Quéau, and H. Rème, Ultra low frequency waves at the magnetic pile-up boundary of comet p/halley, Adv. Space Res., 11, (9)73-(9)77, 1991.

McKean, M. E., D. Winske, and S. P. Gary, Kinetic properties of mirror waves in magnetosheath plasmas, Geophys. Res. Lett., 19, 1331-1334, 1992a.

McKean, M. E., D. Winske, and S. P. Gary, Mirror and ion-cyclotron anisotropy instabilities in the magnetosheath, J. Geophys. Res., 97, 19421-19432, 1992b.

McKean, M. E., S. P. Gary, and D. Winske, Kinetic physics of the mirror instability, J. Geophys. Res., 98, 21313-21321, 1993.

McKean, M. E., D. Winske, and S. P. Gary, 2-dimensional simulations of ion anisotropy instabilities in the magnetosheath, J. Geophys. Res., 99, 11141-11153, 1994.

McKean, M. E., N. Omidi, and D. Krauss-Varban, Wave and ion evolution downstream of quasi-perpendicular bow shocks, J. Geophys. Res., 100, 3427-3437, 1995.

Neubauer, F. M., and K.-H. Glassmeier, Use of an array of satellites as a wave telescope, J. Geophys. Res., 95, 19115-19112, 1990.

Omidi, N., and D. Winske, Structure of the magnetopause inferred from one-dimensional hybrid simulations, J. Geophys. Res., 100, $11935-11955,1995$

Omidi, N., A. O'Farrell, and D. Krauss-Varban, Sources of magnetosheath waves and turbulence, Adv. Space Res., 14, 45-54, 1994.

Onsager, T. G., and M. F. Thomsen, The Earth's foreshock, bow shock, and magnetosheath, Rev. Geophys., 29, 998-1007, 1991.

Pantellini, F. G. E., and S. J. Schwartz, Electron-temperature effects in the linear proton mirror instability, J. Geophys. Res., 100, 3539-3549, 1995.

Pantellini, F. G. E., D. Burgess, and S. J. Schwartz, Phase-space evolution in linear instabilities, Phys. Plasmas, 1, 3784-3791, 1994.
Pantellini, F. G. E., D. Burgess, and S. J. Schwartz, On the non-linear mirror instability, Adv. Space Res., 15, 341-344, 1995.

Paschmann, G., N. Sckopke, S. J. Bame, and J. T. Gosling, Observations of gyrating ions in the foot of the nearly perpendicular bow shock, Geophys. Res. Lett., 9, 881-884, 1982.

Paschmann, G., G. Haerendel, N. Sckopke, E. Mobius, H. Luhr, and C. W. Carlson, 3-dimensional plasma structures with anomalous flow directions near the Earth's bow shock, J. Geophys. Res., 93, 11279-11294, 1988

Phan, T. D., G. Paschmann, W. Baumjohann, N. Sckopke, and H. Lühr, The magnetosheath region adjacent to the dayside magnetopause - AMPTE/IRM observations, J. Geophys. Res. 99, 121-141, 1994.

Phillips, J. L., S. J. Bame, M. F. Thomsen, B. E. Goldstein, and E. J. Smith, Ulysses plasma observations in the Jovian magnetosheath, J. Geophys. Res., 98, 21189-21202, 1993.

Pinçon, J. L., Three-dimensional electromagnetic structure analysis of homogeneous turbulence in space plasma via multipoint measurements by using $k$-filtering, Proceedings of the START ESA Workshop, Aussois, France, 31 January-5 February 1993 , ESA WWP-047, 217-221, 1993.

Pinçon, J. L., and F. Lefeuvre, Local characterization of homogeneous turbulence in a space plasma from simultaneous measurements of field components at several points in space, J. Geophys Res., 96, 11789-11802, 1991.

Rönmark, K., WHAMP - waves in homogeneous, anisotropic multicomponent plasmas, Kiruna Geophys. Inst., Kiruna, Sweden, Report, 179, 1982.

Rose, D. J., General criterion for mirror instability of a plasma, Phys. Fluids, 8, 951-955, 1965,

Russell, C. T., and R. C. Elphic, Initial ISEE magnetometer results: magnetopause observations, Space Sci. Rev., 22, 681, 1978.

Russell, C. T., W. Riedler, K. Schwingenshuh, and Y. Yeroshenko, Mirror instability in the magnetosphere of Comet Halley, Geophys. Res. Lett., 14, 644, 1987.

Russell, C. T., P. Song, and R. P. Lepping, The Uranian magnetopause - lessons from Earth, Geophys. Res. Lett., 16, 1485-1488, 1989.

Schwartz, S. J., Magnetic field structures and related phenomena at quasi-parallel shocks, Adv. Space Res., 11, (9)231-(9)240, 1991.

Schwartz, S. J., Hot flow anomalies near the Earth's bow shock, $A d v$. Space. Res., 15, (8/9)107-(8/9)116, 1995.

Schwartz, S. J., and D. Burgess, Quasi-parallel shocks - a patchwork, of 3-dimensional structures, Geophys. Res. Lett., 18, 373-376, 1991.

Schwartz, S. J., R. L. Kessel, C. C. Brown, L. J. C. Woolliscroft, M. W. Dunlop, C. J. Farrugia, and D. S. Hall, Active current sheets near the Earth's bow shock, J. Geophys. Res., 93, 11295-11310, 1988.

Sckopke, N., G. Paschmann, S. J. Bame, J. T. Gosling, and C. T. Russell, Evolution of ion distributions across the nearly perpendicular bow shock - specularly and non-specularly reflectedgyrating ions, J. Geophys. Res., 88, 6121-6136, 1983.

Sibeck, D. G., Transient events in the outer magnetosphere - boundary waves or flux-transfer events, J. Geophys. Res., 97, 4009-4026, 1992.

Sibeck, D. G., W. Baumjohann, and R. E. Lopez, Solar-wind dynamic pressure variations and transient magnetospheric signatures, Geophys. Res. Lett., 16, 13-16, 1989.

Smith, E. J., and B. T. Tsurutani, Magnetosheath lion roars, J. Geophys. Res., 81, 2261, 1976.

Song, P., ISEE observations of the dayside magnetosheath, Adv Space Res., 14, 71-80, 1994.

Song, P., C. T. Russell, and M. F. Thomsen, Slow mode transition in the frontside magnetosheath, J. Geophys. Res., 97, 8295-8305, 1992a.

Song, P., C. T. Russell, and M. F. Thomsen, Waves in the inner magnetosheath - a case-study, Geophys. Res. Lett., 19 , 2191-2194, 1992b.

Song, P., C. T. Russell, R. J. Fitzenreiter, J. T. Gosling, M. F. Thomsen, D. G. Mitchell, S. A. Fuselier, G. K. Parks, R. R. Anderson, and D. Hubert, Structure and properties of the subsolar 
magnetopause for northward interplanetary magnetic-field - multiple-instrument particle observations, J. Geophys. Res., 98, 11319-11337, 1993

Song, P., G. Le, and C. T. Russell, Observational differences between flux-transfer events and surface-waves at the magnetopause, J. Geophys. Res., 99, 2309-2320, 1994a.

Song, P., C. T. Russell, and S. P. Gary, Identification of low-frequency fluctuations in the terrestrial magnetosheath, J. Geophys. Res., 99, 6011-6025, 1994b.

Song, P., C. T. Russell, X. X. Zhang, S. S. Stahara, and J. R. Spreiter, Boundary layers in the flow at magnetized plasma around a planetary obstacle: the role of the magnetic field, 1995, preprint.

Southwood, D. J., and M. G. Kivelson, On the form of the flow in the magnetosheath, J. Geophys. Res., 97, 2873-2879, 1992.

Southwood, D. J., and M. G. Kivelson, Mirror instability 1. Physical mechanism of linear instability, J. Geophys. Res., 98, 9181-9187, 1993.

Stix, T. H., Waves in Plasmas, American Institute of Physics, New York, 1992.

Stringer, T. E., Low-frequency waves in an unbounded plasma, Plasma Phys. (J. Nucl. Energy), Part C, 5, 89-107, 1963.

Thomsen, M. F., J. T. Gosling, S. J. Bame, K. B. Quest, C. T. Russell, and S. A. Fuselier, On the origin of hot diamagnetic cavities near the Earth's bow shock, J. Geophys. Res., 93, 11311-11325, 1988a.

Thomsen, M. F., J. T. Gosling, and C. T. Russell, ISEE studies of the quasi-parallel bow shock, Adv. Space. Res., 8, (9)175-(9)178, 1988b.

Thomsen, M. F., J. T. Gosling, S. J. Bame, T. G. Onsager, and C. T. Russell, 2-state ion heating at quasi-parallel shocks, J. Geophys. Res., 95, 6363-6374, 1990a.

Thomsen, M. F., J. T. Gosling, S. J. Bame, and C. T. Russell, Magnetic pulses at the quasi-parallel shock, J. Geophys. Res., 95, 957-966, 1990b.
Tsurutani, B. T., E. J. Smith, R. R. Anderson, K. W. Ogilvie, J. D. Scudder, D. N. Baker, and S. J. Bame, Lion roars and nonoscillatory drift mirror waves in the magnetosheath, J. Geophys. Res., 87, 6060-6072, 1982.

Tsurutani, B. T., I. G. Richardson, R. P. Lepping, R. D. Zwickl, D. E. Jones, E. J. Smith, and S. J. Bame, Drift mirror mode waves in the distant $\left(X \approx 200 R_{e}\right)$ magnetosheath, Geophys. Res. Lett., 11, $1102-1105,1984$

Tsurutani, B. T., D. J. Southwood, E. J. Smith, and A. Balogh, Nonlinear magnetosonic waves and mirror mode structures in the March 1991 Ulysses interplanetary event, Geophys. Res. Lett., 19, 1267-1270, 1992.

Violante, L., M. B. Bavassano Cattaneo, G. Morena, and J. D. Richardson, Correction of Observations of mirror waves and plasma depletion layer upstream of Saturn's magnetopause, J. Geophys. Res., 100, 19571, 1995a.

Violante, L., M. B. Bavassano Cattaneo, G. Moreno, and J. D. Richardson, Observations of mirror waves and plasma depletion layer upstream of Saturn's magnetopause, J. Geophys. Res., 100, 12047-12055, 1995b.

vom Stein, R., K.-H. Glassmeier, and U. Motschmann, Cluster as a wave telescope and a mode filter, Proceedings of the ST ART ESA Workshop, Aussois, France, 31 January-5 February 1993, ESA WWP-047, 211-215, 1993.

Winterhalter, D., M. Neugebauer, B. E. Goldstein, E. J. Smith, S. J. Bame, and A. Balogh, Ulysses field and plasma observations of magnetic holes in the solar-wind and their relation to mirror-mode structures, J. Geophys. Res., 99, 23371-23381, 1994 ,

Yan, M., and L. C. Lee, Generation of slow-mode waves in front of the dayside magnetopause, Geophys. Res. Lett., 21, 629-632, 1994.

Zwan, B. J., and R. A. Wolf, Depletion of solar wind plasma near a planetary boundary, J. Geophys. Res., 81, 1636-1648, 1976 